\title{
The Discovery and Mass Measurement of a New Ultra-short-period Planet: EPIC 228732031b
}

Fei Dai ${ }^{1,2}$ (10, Joshua N. Winn ${ }^{2}$ (1), Davide Gandolfi ${ }^{3}$ (1), Sharon X. Wang ${ }^{4}$, Johanna K. Teske ${ }^{5,30}$, Jennifer Burt ${ }^{1}$ (1), Simon Albrecht ${ }^{6}$, Oscar Barragán $^{3}$, William D. Cochran ${ }^{7}$ (10), Michael Endl ${ }^{7}$ (i), Malcolm Fridlund ${ }^{8,9}$ (1), Artie P. Hatzes ${ }^{10}$, Teruyuki Hirano ${ }^{11}$,

Lea A. Hirsch ${ }^{12}$, Marshall C. Johnson ${ }^{13}$ (1) , Anders Bo Justesen ${ }^{6}$, John Livingston ${ }^{14}$, Carina M. Persson ${ }^{9}$, Jorge Prieto-Arranz ${ }^{15,16}$, Andrew Vanderburg ${ }^{17}$ (1) , Roi Alonso ${ }^{15,16}$, Giuliano Antoniciello ${ }^{18}$, Pamela Arriagada ${ }^{4}$ (D), R. P. Butler ${ }^{4}$ (i), Juan Cabrera ${ }^{19}$ (10), Jeffrey D. Crane ${ }^{5}$ (1) , Felice Cusano ${ }^{20}$ (1), Szilárd Csizmadia ${ }^{19}$, Hans Deeg ${ }^{15,16}$ (1), Sergio B. Dieterich ${ }^{4,31}$, Philipp Eigmüller ${ }^{19}$, Anders Erikson $^{19}$, Mark E. Everett ${ }^{21}$ (1), Akihiko Fukui ${ }^{22}$ (1), Sascha Grziwa ${ }^{23}$, Eike W. Guenther ${ }^{10}$, Gregory W. Henry ${ }^{24}$ (1),

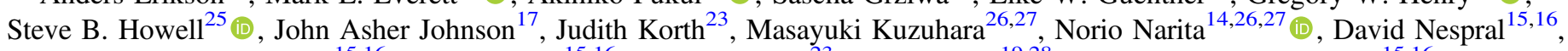
Grzegorz Nowak $^{15,16}$ (D) Enric Palle ${ }^{15,16}$, Martin Pätzold ${ }^{23}$, Heike Rauer ${ }^{19,28}$, Pilar Montañés Rodríguez ${ }^{15,16}$,

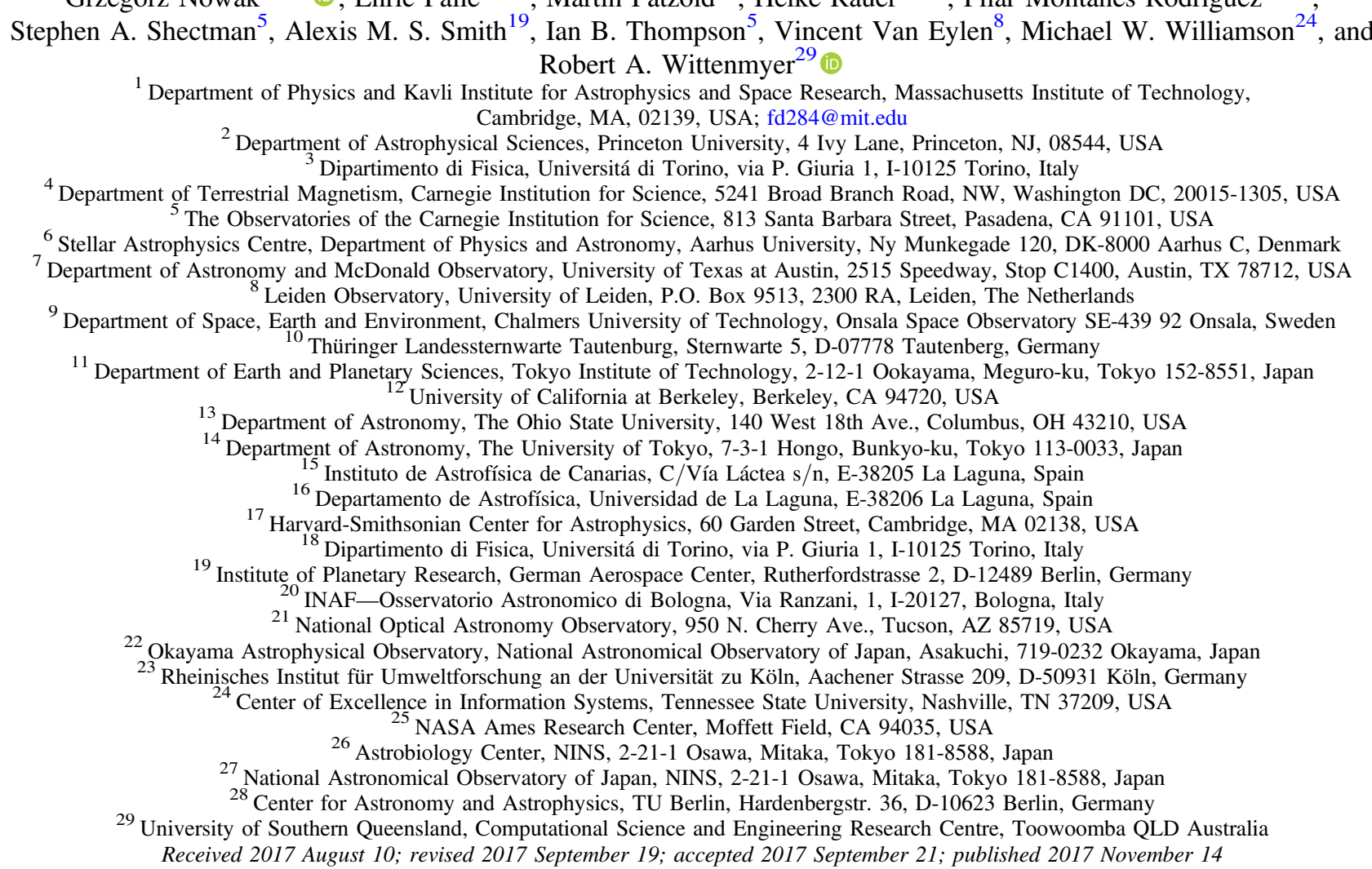

\begin{abstract}
We report the discovery of a new ultra-short-period planet and summarize the properties of all such planets for which the mass and radius have been measured. The new planet, EPIC 228732031b, was discovered in $K 2$ Campaign 10. It has a radius of $1.81_{-0.12}^{+0.16} R_{\oplus}$ and orbits a G dwarf with a period of $8.9 \mathrm{hr}$. Radial velocities obtained with Magellan/PFS and TNG/HARPS-N show evidence for stellar activity along with orbital motion. We determined the planetary mass using two different methods: (1) the "floating chunk offset" method, based only on changes in velocity observed on the same night; and (2) a Gaussian process regression based on both the radial velocity and photometric time series. The results are consistent and lead to a mass measurement of $6.5 \pm 1.6 M_{\oplus}$ and a mean density of $6.0_{-2.7}^{+3.0} \mathrm{~g} \mathrm{~cm}^{-3}$.
\end{abstract}

Key words: planetary systems - stars: individual (EPIC 228732031)

Supporting material: machine-readable tables

\footnotetext{
${ }^{30}$ Carnegie Origins Fellow, jointly appointed by Carnegie DTM and Observatories.

${ }^{31}$ NSF Astronomy and Astrophysics Postdoctoral Fellow.
}

\section{Introduction}

The ultra-short-period (USP) planets, with orbital periods shorter than one day, are usually smaller than about $2 R_{\oplus}$. A well-studied example is Kepler-78b, a roughly Earth-sized planet with an $8.5 \mathrm{hr}$ orbit around a solar-type star (Howard 
et al. 2013; Pepe et al. 2013; Sanchis-Ojeda et al. 2013). Using Kepler data, Sanchis-Ojeda et al. (2014) presented a sample of about 100 transiting USP planets. They found their occurrence rate to be about $0.5 \%$ around G-type dwarf stars, with higher rates for KM stars and a lower rate for F stars. They also noted that many if not all of the USP planets have wider-orbiting planetary companions. It has been postulated that USP planets were once somewhat larger planets that lost their gaseous envelopes (Sanchis-Ojeda et al. 2014; Lopez 2016; Lundkvist et al. 2016; Winn et al. 2017), perhaps after undergoing tidal orbital decay (Lee \& Chiang 2017).

Fulton et al. (2017) reported evidence supporting the notion that planets with a hydrogen-helium $(\mathrm{H} / \mathrm{He})$ envelope can undergo photoevaporation, shrinking their size from 2 to $3 R_{\oplus}$ to $1.5 R_{\oplus}$ or smaller. Specifically, they found the size distribution of close-in $\left(P_{\text {orb }}<100\right.$ days $)$ Kepler planets to be bimodal, with a dip in occurrence between 1.5 and $2 R_{\oplus}$. Owen \& Wu (2013) and Lopez \& Fortney (2014) had predicted such a dip as a consequence of photoevaporation. Owen \& Wu (2017) further demonstrated that the observed radius distribution can be reproduced by a model in which photoevaporation is applied to a single population of super-Earths with gaseous envelopes.

Thus, the USP planets are interesting for further tests and refinements of the photoevaporation theory. They are typically bathed in stellar radiation with a flux $>10^{3}$ higher than the Earth's insolation, where theory predicts they should be rocky cores entirely stripped of $\mathrm{H} / \mathrm{He}$ gas. By studying their distribution in mass, radius, and orbital distance, we may learn about the primordial population of rocky cores and the conditions in which they formed. So far, though, masses have been measured for only a handful of USP planets. The main limitation has been the relative faintness of their host stars, which are drawn mainly from the Kepler survey.

In this paper, we present the discovery and Doppler mass measurement of another USP planet, EPIC 228732031b. The host star is a G-type dwarf with $V=12.1$ that was observed in $K 2$ Campaign 10. This paper is organized as follows. Section 2 presents time-series photometry of EPIC 228732031, both space-based and ground-based. Section 3 describes our radial velocity (RV) observations. Section 4 presents high angular resolution images of the field surrounding EPIC 228732031 and the resultant constraints on any nearby companions. Section 5 is concerned with the stellar parameters of EPIC 228732031, as determined by spectroscopic analysis and stellar evolutionary models. Section 6 presents an analysis of the time-series photometry, including the transit detection, light-curve modeling, and measurement of the stellar rotation period. Section 7 describes the two different methods we employed to analyze the RV data. Section 8 summarizes the properties of all the known USP planets for which mass and radius have been measured.

\section{Photometric Observations}

\section{1. $K 2$}

EPIC 228732031 was observed by the Kepler spacecraft from 2016 July 6 to September 20, during K2 Campaign 10. According to the $K 2$ Data Release Notes, ${ }^{32}$ there was a 3.5-pixel pointing error during the first 6 days of Campaign

\footnotetext{
32 https://keplerscience.arc.nasa.gov/k2-data-release-notes.html
}

10, degrading the data quality. We discarded the data obtained during this period. Later in Campaign 10, the loss of Module 4 resulted in a 14-day gap in data collection. Therefore, the light curves consist of an initial interval of about 6 days, followed by the 14-day data gap, and another continuous interval of about 50 days.

To produce the light curve, we downloaded the target pixel files from the Mikulski Archive for Space Telescopes. ${ }^{33} \mathrm{We}$ then attempted to reduce the well-known apparent brightness fluctuations associated with the rolling motion of the spacecraft, adopting an approach similar to that described by Vanderburg \& Johnson (2014). For each image, we laid down a circular aperture around the brightest pixel and fitted a twodimensional Gaussian function to the intensity distribution. We then fitted a piecewise linear function between the observed flux variation and the central coordinates of the Gaussian function. Figure 1 shows the detrended $K 2$ light curve of EPIC 228732031.

\subsection{Automated Imaging Telescope}

Since the $K 2$ light curve showed signs of stellar activity (as discussed in Section 6), we scheduled ground-based photometric observations of EPIC 228732031, overlapping in time with our RV follow-up campaign. Our hope was that the observed photometric variability could be used to disentangle the effects of stellar activity and orbital motion.

We observed EPIC 228732031 nightly with the Tennessee State University Celestron 14 inch (C14) Automated Imaging Telescope (AIT) located at Fairborn Observatory, Arizona (see, e.g., Henry 1999). The observations were made in the Cousins $R$ bandpass. Each nightly observation consisted of 4-10 consecutive exposures of the field centered on EPIC 228732031. The nightly observations were corrected for bias, flat-fielding, and differential atmospheric extinction. The individual reduced frames were coadded, and aperture photometry was carried out on each coadded frame. We performed ensemble differential photometry: the mean instrumental magnitude of the six comparison stars was subtracted from the instrumental magnitude of EPIC 228732031. Table 1 provides the 149 observations that were collected between 2017 March 15 and May 2.

\subsection{Swope}

EPIC 228732031 was monitored for photometric variability in the Bessel $V$ band from 2017 March 21 to April 1 using the Henrietta Swope $1 \mathrm{~m}$ telescope at Las Campanas Observatory. Exposures of $25 \mathrm{~s}$ were taken consecutively for $2 \mathrm{hr}$ at the beginning and the end of each night if weather permitted. The field of view of the images was $28^{\prime \prime} \times 28^{\prime \prime}$. Initially we selected 59 stars as candidate reference stars for differential aperture photometry. The differential light curve of each star was obtained by dividing the flux of each star by the sum of the fluxes of all the reference stars. The candidate reference stars were then ranked in order of increasing variability. Light curves of EPIC 228732031 were calculated using successively larger numbers of these rank-ordered reference stars. The noise level was found to be minimized when the 16 top-ranked candidate reference stars were used; this collection of stars was adopted to produce the final light curve of EPIC 228732031. Since we

\footnotetext{
33 https://archive.stsci.edu/k2
} 


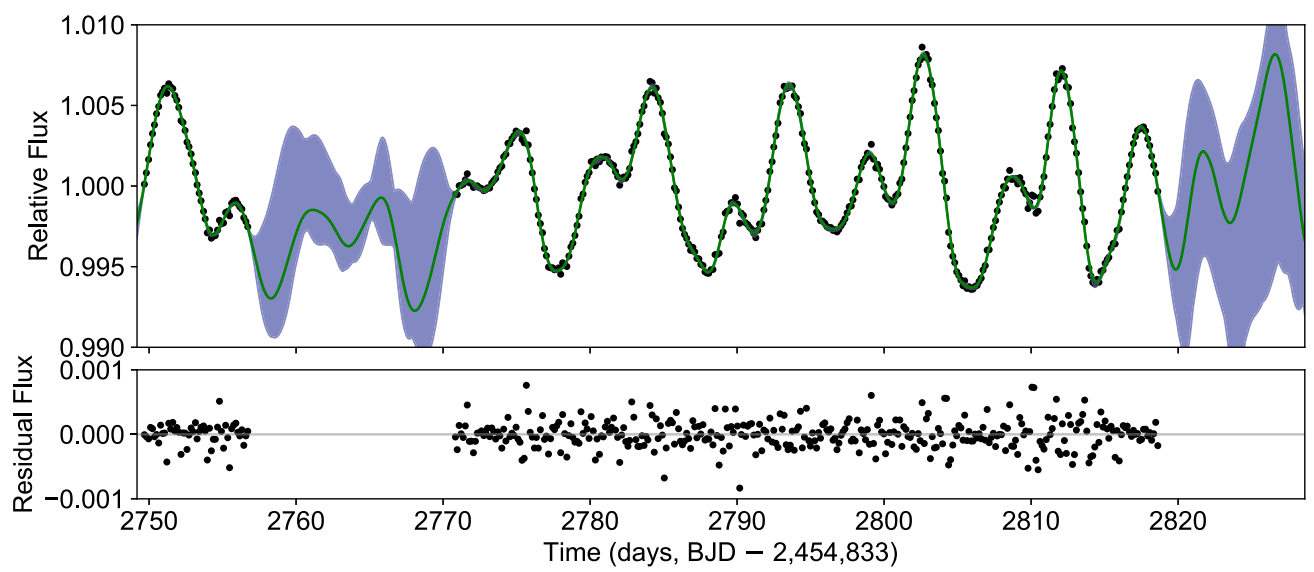

Figure 1. $K 2$ light curve of EPIC 228732031 after removing the transits of planet $b$. The black circles are binned fluxes. The light curve shows a rotational modulation with a period of 9.4 days and an amplitude of about $0.5 \%$. The green curve shows the Gaussian process regression of the $K 2$ light curve with a quasi-periodic kernel (Section 7.2). The blue shaded region is the $1 \sigma$ confidence interval of the Gaussian process.

Table 1

AIT Photometry

\begin{tabular}{lll}
\hline \hline Barycentric Julian Date $\left(\mathrm{BJD}_{\mathrm{TDB}}\right)$ & \multicolumn{1}{c}{$\Delta R$} & Unc \\
\hline 2457827.69557 & -0.92694 & 0.00264 \\
2457827.73587 & -0.9255 & 0.00707 \\
2457827.80667 & -0.93827 & 0.00153 \\
2457827.85367 & -0.93305 & 0.00046 \\
2457827.89417 & -0.93372 & 0.00144 \\
2457827.93727 & -0.93393 & 0.00145 \\
\multicolumn{1}{c}{$\ldots$} & & \\
\hline
\end{tabular}

(This table is available in its entirety in machine-readable form.)

are interested in the long-term variability, we binned the $25 \mathrm{~s}$ exposures taken within each $2 \mathrm{hr}$ window. The relative flux measurements and uncertainties are provided in Table 2.

\section{Radial Velocity Observations}

\subsection{HARPS-N}

Between 2017 January 29 and April 1 (UT), we collected 41 spectra of EPIC 228732031 using the HARPS-N spectrograph $(R \approx 115,000$; Cosentino et al. 2012) mounted on the $3.58 \mathrm{~m}$ Telescopio Nazionale Galileo (TNG) of Roque de los Muchachos Observatory, in La Palma. The observations were carried out as part of the observing programs A33TAC_15 and A33TAC_11. We set the exposure time to $1800-2400 \mathrm{~s}$ and obtained multiple spectra per night. The data were reduced using the HARPS-N off-line pipeline. RVs were extracted by cross-correlating the extracted échelle spectra with a K0 numerical mask (Pepe et al. 2002). Table 3 reports the time of observation, $\mathrm{RV}$, internally estimated measurement uncertainty, FWHM, and bisector span (BIS) of the crosscorrelation function ( $\mathrm{CCF}$ ), the $\mathrm{Ca}$ II $\mathrm{H} \& \mathrm{~K}$ chromospheric activity index $\left(\log R_{\mathrm{HK}}^{\prime}\right)$, the corresponding uncertainties $\left(\Delta \log R_{\mathrm{HK}}^{\prime}\right)$, and the signal-to-noise ratio $(\mathrm{S} / \mathrm{N})$ per pixel at $5500 \AA$.

\subsection{Planet Finder Spectrograph}

We also observed EPIC 228732031 between 2017 March 16 and April 5 (UT), with the Carnegie Planet Finder
Table 2

Swope Photometry

\begin{tabular}{lcr}
\hline \hline BJD $_{\text {TDB }}$ & Relative Flux & Unc. \\
\hline 2457834.08327 & 0.9965 & 0.0035 \\
2457834.35326 & 0.9972 & 0.0041 \\
2457835.10169 & 0.9979 & 0.0046 \\
$\ldots$ & & \\
\hline
\end{tabular}

(This table is available in its entirety in machine-readable form.)

Spectrograph (PFS; $R \approx 76,000$, Crane et al. 2010) on the $6.5 \mathrm{~m}$ Magellan/Clay Telescope at Las Campanas Observatory, Chile. We adopted a similar strategy of obtaining multiple observations during each night. We took two consecutive frames for each visit and attempted three to five visits per night. We obtained a total of 32 spectra in six nights. The detector was read out in the $2 \times 2$ binned mode. The exposure time was set to $1200 \mathrm{~s}$. We obtained a separate spectrum with higher resolution and $\mathrm{S} / \mathrm{N}$, without the iodine cell, to use as a template spectrum. The RVs were determined with the technique of Butler et al. (1996). The internal measurement uncertainties were estimated from the scatter in the results to fitting individual $2 \AA$ sections of the spectrum. The uncertainties ranged from 3 to $6 \mathrm{~m} \mathrm{~s}^{-1}$. Table 4 gives the time of observation, RV, internally estimated measurement uncertainty, and the Ca II $\mathrm{H} \& \mathrm{~K}$ chromospheric activity indicator $S_{\mathrm{HK}}$.

\section{High Angular Resolution Imaging}

\subsection{Speckle Imaging}

On the night of 2017 April 5 (UT), we observed EPIC 228732031 with the NASA Exoplanet Star and Speckle Imager (NESSI), as part of an approved NOAO observing program (PI Livingston, proposal ID 2017A-0377). NESSI is a new instrument for the $3.5 \mathrm{~m}$ WIYN Telescope (N. J. Scott et al. 2017, 2017, in preparation). It uses high-speed electronmultiplying CCDs to capture sequences of $40 \mathrm{~ms}$ exposures simultaneously in two bands: a "blue" band centered at $562 \mathrm{~nm}$ with a width of $44 \mathrm{~nm}$, and a "red" band centered at $832 \mathrm{~nm}$ with a width of $40 \mathrm{~nm}$. We also observed nearby 
Table 3

HARPS-N Observations

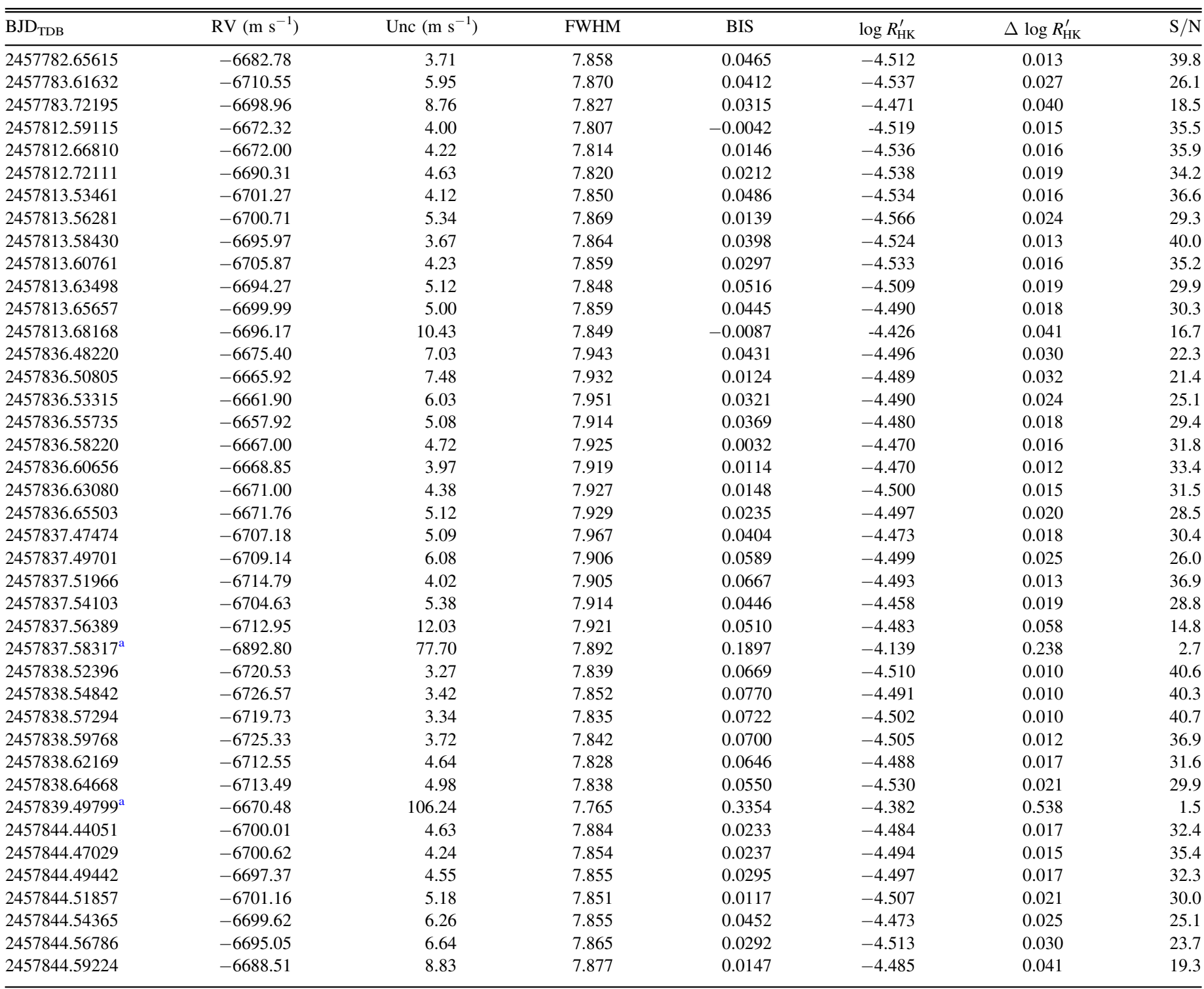

${ }^{a}$ Excluded from analysis due to bad seeing.

point-source calibrator stars close in time. We conducted all observations in the two bands simultaneously. Using the point-source calibrator images, we reconstructed $256 \times 256$ pixel images in each band, corresponding to 4 "! $6 \times 4$ " 6 . No secondary sources were detected in the reconstructed images. We could exclude companions brighter than $3 \%$ and $1 \%$ of the target star respectively in the blue and red band at a separation of $1^{\prime \prime}$. We measured the background sensitivity of the reconstructed images using a series of concentric annuli centered on the target star, resulting in $5 \sigma$ sensitivity limits as a function of angular separation. The resultant contrast curves are plotted in Figure 2.

\subsection{Adaptive Optics}

On the night of 2017 May 23 (UT), we performed adaptive optics (AO) imaging of EPIC 228732031 with the Infrared Camera and Spectrograph (IRCS; Kobayashi et al. 2000) mounted on the $8.2 \mathrm{~m}$ Subaru Telescope. To search for nearby faint companions around EPIC 228732031, we obtained lightly saturated frames using the $H$-band filter with individual exposure times of $10 \mathrm{~s}$. We coadded the exposures in groups of three. The observations were performed in the highresolution mode ( 1 pixel $=20.6$ mas) using five-point dithering to minimize the impact of bad and hot pixels. We repeated the integration sequence for a total exposure time of $300 \mathrm{~s}$. For absolute flux calibration, we also obtained unsaturated frames in which the individual exposure time was set to $0.412 \mathrm{~s}$ and coadded three exposures.

We reduced the IRCS raw data as described by Hirano et al. (2016). We applied bias subtraction, flat-fielding, and distortion corrections before aligning and median-combining each of the saturated and unsaturated frames. The FWHM of the combined unsaturated image was $00^{\prime \prime} 10$. The combined saturated image exhibits no bright source within the field of view of 21 !" $0 \times 21$." 0 . To estimate the achieved flux contrast, we 
Table 4

PFS Observations

\begin{tabular}{|c|c|c|c|}
\hline$\overline{\mathrm{BJD}_{\mathrm{TDB}}}$ & $\mathrm{RV}\left(\mathrm{m} \mathrm{s}^{-1}\right)$ & Unc $\left(\mathrm{m} \mathrm{s}^{-1}\right)$ & $\overline{\mathrm{S}_{\mathrm{HK}}}$ \\
\hline 2457828.85718 & -45.17 & 3.88 & 0.701 \\
\hline 2457828.87266 & -36.47 & 4.03 & 0.658 \\
\hline 2457829.70652 & -20.52 & 3.55 & 0.534 \\
\hline 2457829.72141 & -4.58 & 6.07 & 0.618 \\
\hline 2457830.59287 & 17.71 & 3.56 & 0.552 \\
\hline 2457830.60890 & 23.34 & 3.48 & 0.492 \\
\hline 2457830.68486 & 21.34 & 3.51 & 0.478 \\
\hline 2457830.70112 & 19.92 & 3.26 & 0.498 \\
\hline 2457830.74201 & -0.74 & 3.44 & 0.474 \\
\hline 2457830.75830 & 16.90 & 3.50 & 0.489 \\
\hline 2457830.83794 & 8.25 & 4.64 & 0.503 \\
\hline 2457830.85420 & -4.06 & 4.91 & 0.803 \\
\hline 2457832.60032 & -29.51 & 4.96 & 0.635 \\
\hline 2457832.61610 & -21.56 & 4.11 & 0.603 \\
\hline 2457832.69542 & -25.76 & 5.59 & 0.693 \\
\hline $2457832.71105^{\mathrm{a}}$ & -51.72 & 10.95 & 0.742 \\
\hline 2457833.62080 & 2.10 & 3.91 & 0.596 \\
\hline 2457833.63700 & 4.38 & 3.73 & 0.595 \\
\hline 2457833.71138 & 2.23 & 4.12 & 0.527 \\
\hline 2457833.72778 & -1.98 & 4.06 & 0.539 \\
\hline 2457833.82991 & 0.55 & 3.54 & 0.502 \\
\hline 2457833.84582 & -4.49 & 3.69 & 0.560 \\
\hline 2457848.53428 & 9.40 & 4.49 & 0.668 \\
\hline 2457848.55023 & 2.09 & 4.17 & 0.658 \\
\hline 2457848.63414 & 7.27 & 4.23 & 0.687 \\
\hline 2457848.65008 & 6.40 & 4.15 & 0.562 \\
\hline 2457848.70933 & 29.22 & 3.92 & 0.492 \\
\hline 2457848.72551 & 14.37 & 4.19 & 0.572 \\
\hline 2457848.75682 & 23.83 & 5.51 & 0.616 \\
\hline 2457848.77330 & 6.85 & 5.44 & 0.562 \\
\hline 2457848.79458 & 19.56 & 5.14 & 0.437 \\
\hline 2457848.81068 & 10.63 & 5.45 & 0.885 \\
\hline
\end{tabular}

${ }^{a}$ Excluded from analysis due to bad seeing.

convolved the combined saturated image with a kernel having a radius equal to half the FWHM. We then computed the scatter as a function of radial separation from EPIC 228732031. Figure 3 shows the resulting $5 \sigma$ contrast curve, along with a zoomed-in image of EPIC 228732031 with a field of view of 4 ." $0 \times 4$." 0 . We can exclude companions brighter than $6 \times 10^{-4}$ of the target star, over separations of $1^{\prime \prime}-4$ !. 0 .

\section{Stellar Parameters}

We determined the spectroscopic parameters of EPIC 228732031 from the coadded HARPS-N spectrum, which has an $\mathrm{S} / \mathrm{N}$ per pixel of about 165 at $5500 \AA$. We used four different methods to extract the spectroscopic parameters:

Method 1. We used the spectral synthesis code SPECTRUM ${ }^{34}$ (V2.76; Gray \& Corbally 1994) to compute synthetic spectra using ATLAS 9 model atmospheres (Castelli \& Kurucz 2004). We adopted the calibration equations of Bruntt et al. (2010b) and Doyle et al. (2014) to derive the microturbulent $\left(v_{\text {mic }}\right)$ and macroturbulent $\left(v_{\mathrm{mac}}\right)$ velocities. We focused on spectral features that are most sensitive to varying photospheric parameters. Briefly, we used the wings of the $\mathrm{H} \alpha$ line to obtain an initial estimate of the effective temperature $\left(T_{\text {eff }}\right)$. We then used the $\operatorname{MgI} 5167,5173,5184 \AA$, the $\mathrm{CaI} 6162$,

\footnotetext{
$\overline{34}$ http://www.appstate.edu/ grayro/spectrum/spectrum.html
}
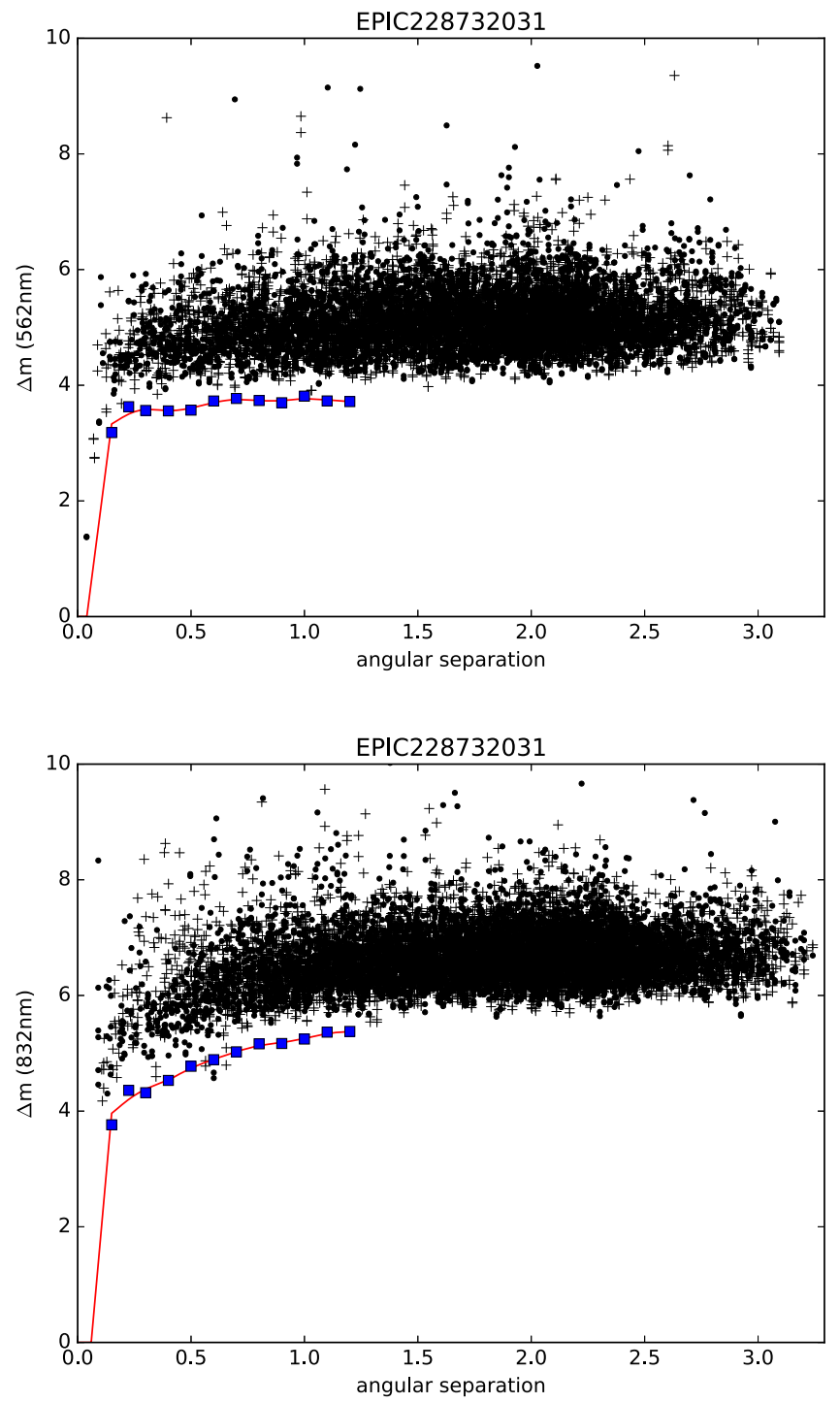

Figure 2. The $5 \sigma$ contrast curve based on the speckle images obtained with WIYN/NESSI. The upper panel shows a "blue" band centered at $562 \mathrm{~nm}$ with a width of $44 \mathrm{~nm}$, and the lower panel shows a "red" band centered at $832 \mathrm{~nm}$ with a width of $40 \mathrm{~nm}$. The blue squares are $5 \sigma$ sensitivity limits as a function of angular separation. No secondary sources were detected in the reconstructed images. The data points represent local extrema measured in the background sky of our reconstructed speckle image. Plus signs are local maxima and dots are local minima. The blue squares show the $5 \sigma$ background sensitivity limit, and the smooth curve is the spline fit.

$6439 \AA$, and the Na I D lines to refine the effective temperature and derive the surface gravity $(\log g)$. The iron abundance $[\mathrm{Fe} / \mathrm{H}]$ and projected rotational velocity $v \sin i_{\star}$ were estimated by fitting many isolated and unblended iron lines. The results were as follows: $T_{\text {eff }}=5225 \pm 70 \mathrm{~K}, \log g=4.67 \pm 0.08$ (cgs), $[\mathrm{Fe} / \mathrm{H}]=0.01 \pm 0.05 \mathrm{dex}, v \sin i_{\star}=4.8 \pm 0.6 \mathrm{~km} \mathrm{~s}^{-1}$, $v_{\text {mic }}=0.86 \pm 0.10 \mathrm{~km} \mathrm{~s}^{-1}$, and $v_{\text {mac }}=2.07 \pm 0.48 \mathrm{~km} \mathrm{~s}^{-1}$.

Method 2. We also determined the spectroscopic parameters using the equivalent-width method. The analysis was carried out with ispec (Blanco-Cuaresma et al. 2014). The effective temperature $T_{\text {eff }}$, surface gravity $\log g$, metallicity $[\mathrm{Fe} / \mathrm{H}]$, and microturbulence $v_{\text {mic }}$ were iteratively determined using $116 \mathrm{Fe} \mathrm{I}$ and $15 \mathrm{Fe}$ II lines by requiring excitation balance, ionization balance, and the agreement between Fe I and Fe II abundances. Synthetic spectra were calculated using MOOG (Sneden 1973) and MARCS model atmospheres (Gustafsson et al. 2008). The 


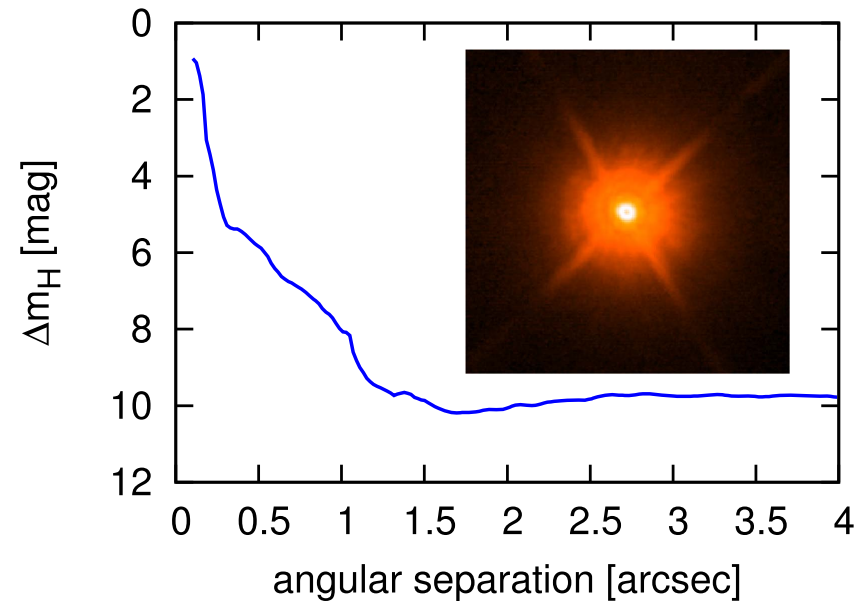

Figure 3. $H$-band $5 \sigma$ contrast curve for EPIC 228732031 based on the saturated image obtained with Subaru/IRCS. The inset displays a $4^{\prime \prime} \times 4^{\prime \prime}$ image of EPIC 228732031. These data exclude companions down to a contrast of $6 \times 10^{-4}$ at a separation of $1^{\prime \prime}$.

projected rotation velocity $v \sin i_{\star}$ was determined by convolving the synthetic spectrum with a broadening kernel to match the observed spectrum. The results were as follows: $T_{\text {eff }}=5216 \pm 27 \mathrm{~K}, \log g=4.63 \pm 0.05(\mathrm{cgs}),[\mathrm{Fe} / \mathrm{H}]=$ $-0.02 \pm 0.09 \mathrm{dex}$, and $v \sin i_{\star}=4.0 \pm 0.6 \mathrm{~km} \mathrm{~s}^{-1}$.

Method 3. We fitted the observed spectrum to theoretical ATLAS12 model atmospheres from Kurucz (2013) using SME version 5.22 (Valenti \& Piskunov 1996; Valenti \& Fischer 2005; Piskunov \& Valenti 2017). ${ }^{35}$ We used the atomic and molecular line data from VALD3 (Piskunov et al. 1995; Kupka \& Ryabchikova 1999). ${ }^{36}$ We used the empirical calibration equations for Sun-like stars from Bruntt et al. (2010a) and Doyle et al. (2014) to determine the microturbulent $\left(v_{\text {mic }}\right)$ and macroturbulent $\left(v_{\mathrm{mac}}\right)$ velocities. The projected stellar rotational velocity $v \sin i_{\star}$ was estimated by fitting about 100 clean and unblended metal lines. To determine the $T_{\text {eff }}$, the $\mathrm{H} \alpha$ profile was fitted to the appropriate model (Fuhrmann et al. 1993; Axer et al. 1994; Fuhrmann et al. 1994, 1997a, 1997b). Then we iteratively fitted for $\log g$ and $[\mathrm{Fe} / \mathrm{H}]$ using the $\mathrm{Ca}$ I lines at $6102,6122,6162$, and $6439 \AA$, as well as the $\mathrm{Na}$ I doublet at 5889.950 and $5895.924 \AA$. The results were as follows: $T_{\text {eff }}=4975 \pm 125 \mathrm{~K}, \log g=4.40 \pm 0.15$ (cgs), [Fe/ $\mathrm{H}]=-0.06 \pm 0.10 \mathrm{dex}$, and $v \sin i_{\star}=4.8 \pm 1.6 \mathrm{~km} \mathrm{~s}^{-1}$.

Method 4. We took a more empirical approach using SpecMatch-emp ${ }^{37}$ (Yee et al. 2017). This code estimates the stellar parameters by comparing the observed spectrum with a library of about 400 well-characterized stars (M5 to F1) observed by Keck/HIRES. SpecMatch-emp gave $T_{\text {eff }}=$ $5100 \pm 110 \mathrm{~K},[\mathrm{Fe} / \mathrm{H}]=-0.06 \pm 0.09$, and $R_{\star}=0.75 \pm$ $0.10 R_{\odot}$. SpecMatch-emp directly yields stellar radius rather than the surface gravity because the library stars typically have their radii calibrated using interferometry and other techniques. With the stellar radius, $T_{\text {eff }}$, and $[\mathrm{Fe} / \mathrm{H}]$, we estimated the surface gravity using the empirical relation by Torres et al. (2010): $\log g=4.60 \pm 0.10$.

The spectroscopic parameters from these four methods do not agree with each other within the quoted uncertainties (summarized in Table 5), even though they are all based on the

\footnotetext{
$\overline{35}$ http://www.stsci.edu/ valenti/sme.html

36 http://vald.astro.uu.se

37 https://github.com/samuelyeewl/specmatch-emp
}

same data. In particular, the effective temperature from Method 3 is about $2 \sigma$ lower than the weighted mean of all the results. This disagreement is typical in studies of this nature and probably arises because the quoted uncertainties do not include systematic effects associated with the different assumptions and theoretical models. For the analysis that follows, we computed the weighted mean of each spectroscopic parameter and assigned it an uncertainty equal to the standard deviation among the four different results. The uncertainties thus derived are likely underestimated because of systematic biases introduced by the various model assumptions that are difficult to quantify. The results are as follows: $T_{\text {eff }}=5200 \pm 100 \mathrm{~K}$, $\log g=4.62 \pm 0.10,[\mathrm{Fe} / \mathrm{H}]=-0.02 \pm 0.08$, and $v \sin i_{\star}=$ $4.4 \pm 1.0 \mathrm{~km} \mathrm{~s}^{-1}$.

We determined the stellar mass and radius using the code Isochrones (Morton 2015). This code takes as input the spectroscopic parameters, as well as the broadband photometry of EPIC 228732031 retrieved from the ExoFOP website. ${ }^{38}$ The various inputs are fitted to the stellar evolutionary models from the Dartmouth Stellar Evolution Database (Dotter et al. 2008). We used the nested sampling code MultiNest (Feroz et al. 2009) to sample the posterior distribution. The results were $M_{\star}=0.84 \pm 0.03 M_{\odot}$ and $R_{\star}=0.81 \pm 0.03 R_{\odot}$.

We derived the interstellar extinction $\left(A_{\mathrm{v}}\right)$ and distance $(d)$ to EPIC 228732031 following the technique described in Gandolfi et al. (2008). Briefly, we fitted the $B-V$ and 2MASS colors using synthetic magnitudes extracted from the NEXTGEN model spectrum (Hauschildt et al. 1999) with the same spectroscopic parameters as the star. Adopting the extinction law of Cardelli et al. (1989) and assuming a total-to-selective extinction of $R_{\mathrm{v}}=3.1$, we found that EPIC 228732031 suffers from a small amount of reddening of $A_{\mathrm{v}}=0.07 \pm 0.05$ mag. Assuming a blackbody emission at the star's effective temperature and radius, we derived a distance from the Sun of $d=174 \pm 20 \mathrm{pc}$.

\section{Photometric Analysis}

\subsection{Transit Detection}

Before searching the $K 2$ light curve for transits, we removed long-term systematic or instrumental flux variations by fitting a cubic spline of length 1.5 days, and then dividing by the spline function. We searched for periodic transit signals using the box-least-squares algorithm (BLS; Kovács et al. 2002). Following the suggestion of Ofir (2014), we employed a nonlinear frequency grid to account for the expected scaling of transit duration with orbital period. We also adopted his definition of signal detection efficiency (SDE), in which the significance of a detection is quantified by first subtracting the local median of the BLS spectrum and then normalizing by the local standard deviation. The transit signal of EPIC 228732031b was detected with an SDE of 14.4.

We searched for additional transiting planets in the system by rerunning the BLS algorithm after removing the data within $2 \mathrm{hr}$ of each transit of planet b. No significant transit signal was detected: the maximum SDE of the new BLS spectrum was 4.5. Visual inspection of the light curve also did not reveal any significant transit events. In particular, no transit was seen at the orbital period of 3.0 days, the period which emerged as the dominant peak in the periodogram of the radial velocity data

\footnotetext{
${ }^{38}$ https://exofop.ipac.caltech.edu
} 
Table 5

Spectroscopic Parameters of EPIC 228732031

\begin{tabular}{|c|c|c|c|c|c|}
\hline Parameters & Method 1 & Method 2 & Method 3 & Method 4 & Adopted \\
\hline$T_{\text {eff }}(K)$ & $5225 \pm 70$ & $5216 \pm 27$ & $4975 \pm 125$ & $5100 \pm 110$ & $5200 \pm 100$ \\
\hline $\log g($ dex $)$ & $4.67 \pm 0.08$ & $4.63 \pm 0.05$ & $4.40 \pm 0.15$ & $4.60 \pm 0.10$ & $4.62 \pm 0.10$ \\
\hline$[\mathrm{Fe} / \mathrm{H}](\mathrm{dex})$ & $0.01 \pm 0.05$ & $-0.02 \pm 0.09$ & $-0.06 \pm 0.10$ & $-0.06 \pm 0.09$ & $-0.02 \pm 0.08$ \\
\hline
\end{tabular}

(See Section 7.3.1). The upper panel of Figure 1 shows the light curve after removing the transits of planet $b$.

\subsection{Transit Modeling}

The orbital period, midtransit time, transit depth, and transit duration from BLS were used as the starting point for a more rigorous transit analysis. We modeled the transit light curves with the Python package Batman (Kreidberg 2015). We isolated the transits using a $4 \mathrm{hr}$ window around the time of midtransit. The free parameters included in the model were the orbital period $P_{\mathrm{orb}}$, the midtransit time $t_{\mathrm{c}}$, the planet-to-star radius ratio $R_{\mathrm{p}} / R_{\star}$, the scaled orbital distance $a / R_{\star}$, and the impact parameter $b \equiv a \cos i / R_{\star}$. We adopted a quadratic limb-darkening profile. We imposed Gaussian priors on the limb-darkening coefficients $u_{1}$ and $u_{2}$ with the median from EXOFAST $^{39}\left(u_{1}=0.52, u_{2}=0.19\right.$, Eastman et al. 2013) and widths of 0.1 . Jeffreys priors were imposed on $P_{\mathrm{orb}}, R_{p} / R_{\star}$, and $a / R_{\star}$. Uniform priors were imposed on $t_{\mathrm{c}}$ and $\cos i$. Since the data were obtained with 30-minute averaging, we sampled the model light curve at 1-minute intervals and then averaged to 30 minutes to account for the finite integration time (Kipping 2010).

We adopted the usual $\chi^{2}$ likelihood function and found the best-fit solution using the Levenberg-Marquardt algorithm implemented in the Python package lmfit. Figure 4 shows the phase-folded light curve and the best-fitting model. In order to test if planet $\mathrm{b}$ displays transit timing variations (TTV), we used the best-fit transit model as a template. We fitted each individual transit, varying only the midtransit time and a quadratic function of time to describe any residual long-term flux variation. The resultant transit times are consistent with a constant period (Figure 5). We proceeded with the analysis under the assumption that any TTVs are negligible given the current sensitivity.

To sample the posterior distribution of various transit parameters, we performed a Markov Chain Monte Carlo (MCMC) analysis with emcee (Foreman-Mackey et al. 2013). We launched 100 walkers in the vicinity of the best-fit solution. We stopped the walkers after running 5000 links and discarded the first 1000 links. Using the remaining links, the GelmanRubin potential scale reduction factor was found to be within 1.03 , indicating adequate convergence. The posterior distributions for all parameters were smooth and unimodal. Table 7 reports the results, based on the $16 \%, 50 \%$, and $84 \%$ levels of the cumulative posterior distribution. The mean stellar density obtained from transit modeling assuming a circular orbit $\left(2.43_{-1.09}^{+0.61} \mathrm{~g} \mathrm{~cm}^{-3}\right)$ agrees with that computed from the mass and radius derived in Section $5\left(2.23 \pm 0.33 \mathrm{~g} \mathrm{~cm}^{-3}\right)$.

\footnotetext{
39 astroutils.astronomy.ohio-state.edu/exofast/limbdark.shtml
}

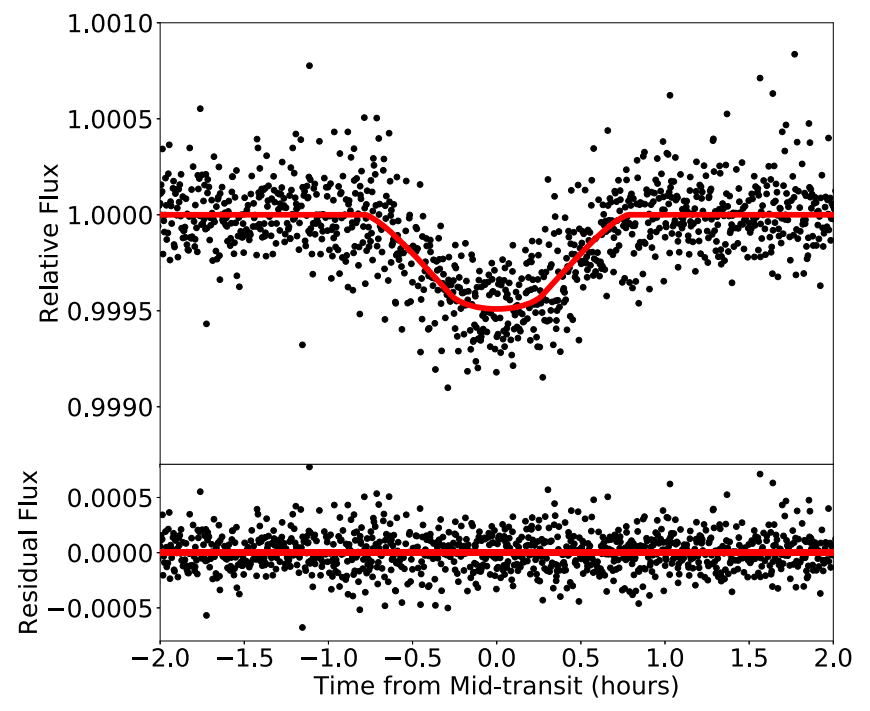

Figure 4. The best-fit transit model of EPIC 228732031b. The black dots are $K 2$ observations. The red line is the best-fit transit model after accounting for the effect of the 30-minute time averaging.

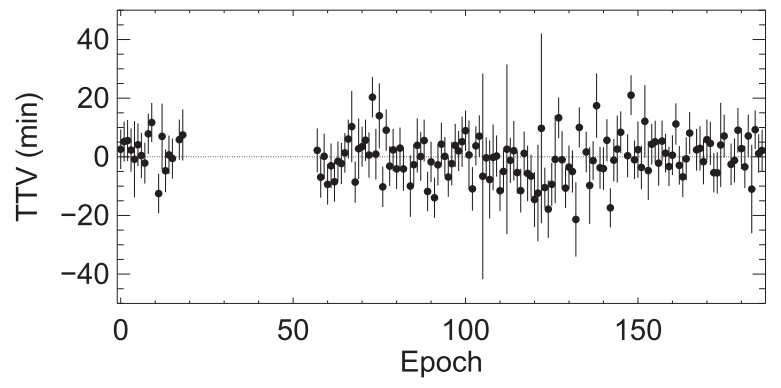

Figure 5. The transit time variations of EPIC $228732031 \mathrm{~b}$ observed by $K 2$. They have large uncertainties due to the combination of a 30-minute time averaging and the short transit duration of $\approx 1 \mathrm{hr}$. The transit times are consistent with a constant period.

\subsection{Stellar Rotation Period}

The $K 2$ light curve showed quasi-periodic modulations that are likely associated with magnetic activity coupled with stellar rotation (see upper panel of Figure 1). To measure the stellar rotation period, we computed the Lomb-Scargle Periodogram (Lomb 1976; Scargle 1982) of the K2 light curve, after removing the transits of planet $b$. The strongest peak is at $9.37 \pm 1.85$ days. Computing the autocorrelation function (McQuillan et al. 2014) leads to a consistent estimate for the stellar rotation period of $9.2_{-1.2}^{+2.3}$ days. Analysis of the groundbased AIT light curve also led to a consistent estimate of $9.84 \pm 0.80$ days (see Figures 6 and 7). The amplitude of the rotationally modulated variability was about $0.5 \%$ in both data sets. 
Table 6

Stellar Parameters of EPIC 228732031

\begin{tabular}{|c|c|c|}
\hline Parameters & Value and $68.3 \%$ Conf. Limits & Reference \\
\hline R.A. $\left({ }^{\circ}\right)$ & 182.751556 & $\mathrm{~A}$ \\
\hline Decl. $\left({ }^{\circ}\right)$ & -9.765218 & A \\
\hline$V(\mathrm{mag})$ & $12.115 \pm 0.020$ & A \\
\hline$T_{\text {eff }}(K)$ & $5200 \pm 100$ & B \\
\hline $\log g(\operatorname{dex})$ & $4.62 \pm 0.10$ & $\mathrm{~B}$ \\
\hline$[\mathrm{Fe} / \mathrm{H}](\mathrm{dex})$ & $-0.02 \pm 0.08$ & B \\
\hline$v \sin i\left(\mathrm{~km} \mathrm{~s}^{-1}\right)$ & $4.4 \pm 1.0$ & $\mathrm{~B}$ \\
\hline$M_{\star}\left(M_{\odot}\right)$ & $0.84 \pm 0.03$ & $\mathrm{~B}$ \\
\hline$R_{\star}\left(R_{\odot}\right)$ & $0.81 \pm 0.03$ & $\mathrm{~B}$ \\
\hline$P_{\text {rot }}$ (days) & $9.37 \pm 1.85$ & B \\
\hline$\rho_{\text {spe }}\left(\mathrm{g} \mathrm{cm}^{-3}\right)^{\mathrm{a}}$ & $2.23 \pm 0.33$ & B \\
\hline$\rho_{\text {tra }}\left(\mathrm{g} \mathrm{cm}^{-3}\right)^{\mathrm{b}}$ & $2.43_{-1.09}^{+0.61}$ & B \\
\hline $\mathrm{u}_{1}$ & $0.53 \pm 0.10$ & B \\
\hline $\mathrm{u}_{2}$ & $0.20 \pm 0.10$ & $\mathrm{~B}$ \\
\hline$A_{\mathrm{v}}(\mathrm{mag})$ & $0.07 \pm 0.05$ & $\mathrm{~B}$ \\
\hline$d(\mathrm{pc})$ & $174 \pm 20$ & B \\
\hline
\end{tabular}

Notes. A: ExoFOP; B: this work.

${ }^{\mathrm{a}}$ Mean density from the derived mass and radius.

b Mean density from modeling the transit light curve.

Using the measured values of $P_{\text {rot }}, R_{\star}$, and $v \sin i_{\star}$, it is possible to check for a large spin-orbit misalignment along the line of sight. Our spectroscopic analysis gave $v \sin i_{\star}=4.4 \pm$ $1.0 \mathrm{~km} \mathrm{~s}^{-1}$. Using the stellar radius and rotation period reported in Table $6, v=2 \pi R_{\star} / P_{\text {rot }}=4.4 \pm 1.1 \mathrm{~km} \mathrm{~s}^{-1}$. Because these two values are consistent, there is no evidence for any misalignment, and the $2 \sigma$ lower limit on $\sin i_{\star}$ is 0.48 .

\section{Radial Velocity Analysis}

Stellar variability is a frequent source of correlated noise in precise RV data. Stellar variability may refer to several effects, including $p$-mode oscillations, granulation, magnetic activity coupled with stellar rotation, and long-term magnetic activity cycles. The most problematic component is often the magnetic activity coupled with stellar rotation. The magnetic activity of a star gives rise to surface inhomogeneities: spots, plages, and faculae. As these active regions are carried around by the rotation of the host star, they produce two major effects on the radial velocity measurement (see, e.g., Lindegren \& Dravins 2003; Haywood et al. 2016). (1) The "rotational" component: stellar rotation carries the surface inhomogeneities from the blueshifted to the redshifted part of the star, distorting the spectral lines and throwing off the apparent radial velocity. (2) The "convective" component: the suppression of convective blueshift in strong magnetic regions leads to a net radial velocity shift whose amplitude depends on the orientation of the surface relative to the observer's line of sight. Both of these effects produce quasi-periodic variations in the radial velocity measurements on the timescale of the stellar rotation period.

The median value of $\log R_{\mathrm{HK}}^{\prime}$ for EPIC 228732031 was -4.50 . This suggests a relatively strong chromospheric activity level, according to Isaacson \& Fischer (2010). For comparison, Egeland et al. (2017) measured a mean $\log R_{\mathrm{HK}}^{\prime}$ of about -4.96 for the Sun during solar cycle 24. According to Fossati et al. (2017), the measured $\log R_{\mathrm{HK}}^{\prime}$ is likely suppressed by the Ca II lines in the interstellar medium. The star might be more active than what the measured $\log R_{\mathrm{HK}}^{\prime}$ suggests. The amplitude of the rotational modulation seen in the photometry is well in excess of the Sun's variability. Figure 8 shows the measured RV, plotted against activity indicators. The different colors represent data obtained on different nights. The data from different nights tend to cluster together in these plots. This implies that the pattern of stellar activity changes on a nightly basis, and that the RVs are correlated with stellar activity. To quantify the significance of the correlations, we applied the Pearson correlation test to each activity indicator. BIS, FWHM, and $S_{\mathrm{HK}}$ showed the strongest correlations with $p$ values of $2.4 \times 10^{-6}, 0.014$, and 0.027 , respectively. Both the PFS and HARPS-N data were affected by correlated noise. In order to extract the planetary signal, we used two different approaches: the "floating chunk offset" method and the Gaussian process regression, as described below.

\subsection{Floating Chunk Offset Method}

The floating chunk offset method (see, e.g., Hatzes et al. 2011) takes advantage of the clear separation of timescales between the orbital period ( 0.37 days) and the stellar rotation period (9.4 days). Only the changes in velocity observed within a given night are used to determine the spectroscopic orbit, and thereby the planet mass. In practice, this is done by fitting all of the data but allowing the data from each night to have an arbitrary RV offset. This method requires multiple observations taken within the same night, such as those presented in this paper.

The PFS and HARPS-N data span 14 nights, thereby introducing 14 parameters: $\gamma_{1}$ to $\gamma_{14}$. We fitted a model in which the orbit was required to be circular, and another model in which the orbit was allowed to be eccentric. The circular model has three parameters: the RV semiamplitude $K$, the orbital period $P_{\text {orb }}$, and the time of conjunction $t_{\mathrm{c}}$. The eccentric model has two additional parameters: the eccentricity $e$ and the argument of periastron $\omega$; for the fitting process, we used $\sqrt{e} \cos \omega$ and $\sqrt{e} \sin \omega$. We also included a separate "jitter" parameter $\sigma_{\text {jit }}$ for PFS and HARPS-N. The jitter parameter accounts for both time-uncorrelated astrophysical RV noise as well as instrumental noise in excess of the internally estimated uncertainty. We imposed Gaussian priors on the orbital period and time of conjunction, based on the photometric results from Section 6.2. We imposed Jeffreys priors on $K$ and $\sigma_{\text {jit }}$ and uniform priors on $\sqrt{e} \cos \omega$ (with range $[-1,1]$ ), $\sqrt{e} \sin \omega$ $([-1,1])$, and $\gamma_{1}$ to $\gamma_{14}$.

We adopted the following likelihood function:

$$
\begin{aligned}
\mathcal{L}= & \prod_{i}\left(\frac{1}{\sqrt{2 \pi\left(\sigma_{i}^{2}+\sigma_{\mathrm{jit}}\left(t_{i}\right)^{2}\right)}}\right. \\
& \left.\times \exp \left[-\frac{\left[\mathrm{RV}\left(t_{i}\right)-\mathcal{M}\left(t_{i}\right)-\gamma\left(t_{i}\right)\right]^{2}}{2\left(\sigma_{i}^{2}+\sigma_{\mathrm{jit}}\left(t_{i}\right)^{2}\right)}\right]\right),
\end{aligned}
$$

where $\mathrm{RV}\left(t_{i}\right)$ is the measured radial velocity at time $t_{i}, \mathcal{M}\left(t_{i}\right)$ is the calculated radial velocity variation at time $t_{i}, \sigma_{i}$ is the internal measurement uncertainty, $\sigma_{\mathrm{jit}}\left(t_{i}\right)$ is the jitter parameter specific to the instrument used, and $\gamma\left(t_{i}\right)$ is the arbitrary RV offset specific to each night.

We obtained the best-fit solution using the LevenbergMarquardt algorithm implemented in the Python package lmfit (see Figure 9). To sample the posterior distribution, we performed an MCMC analysis with emcee following a procedure similar to that described in Section 6.2. Table 7 


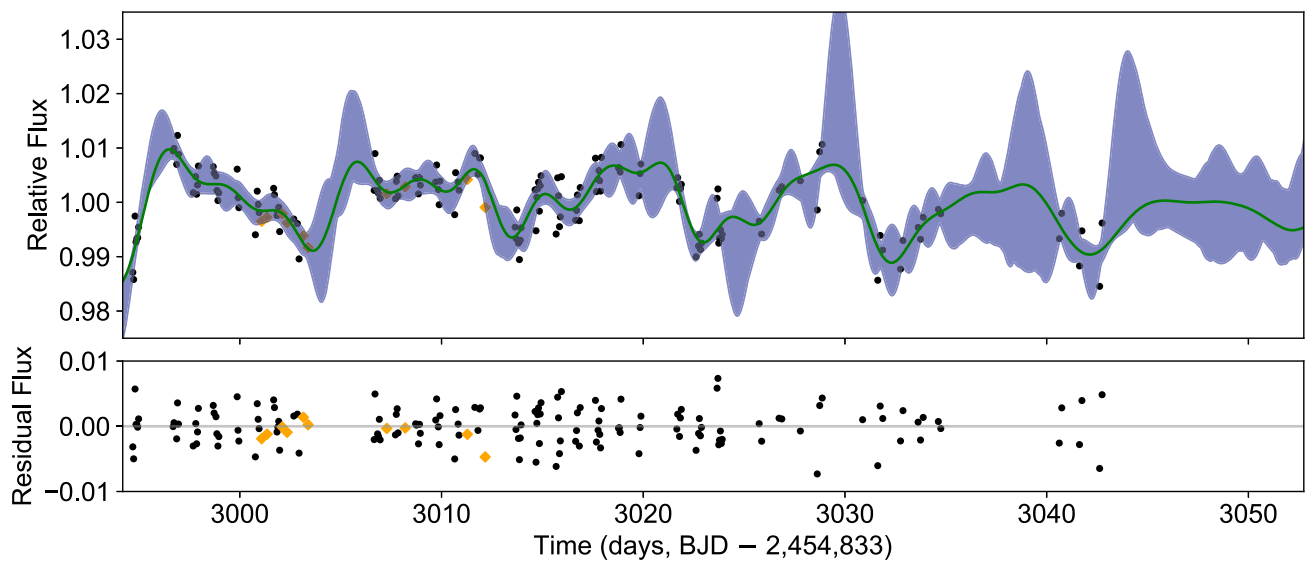

Figure 6. Ground-based light curve of EPIC 228732031. The black circles are observed fluxes from AIT. The orange diamonds are observed fluxes from Swope. The light curve shows a rotational modulation with periodicity similar to that of the $K 2$ light curve (See Figure 7). The green curve shows the Gaussian process regression of the light curve with the same quasi-periodic kernel as in Figure 1. The blue shaded region is the $1 \sigma$ confidence interval of the Gaussian process.

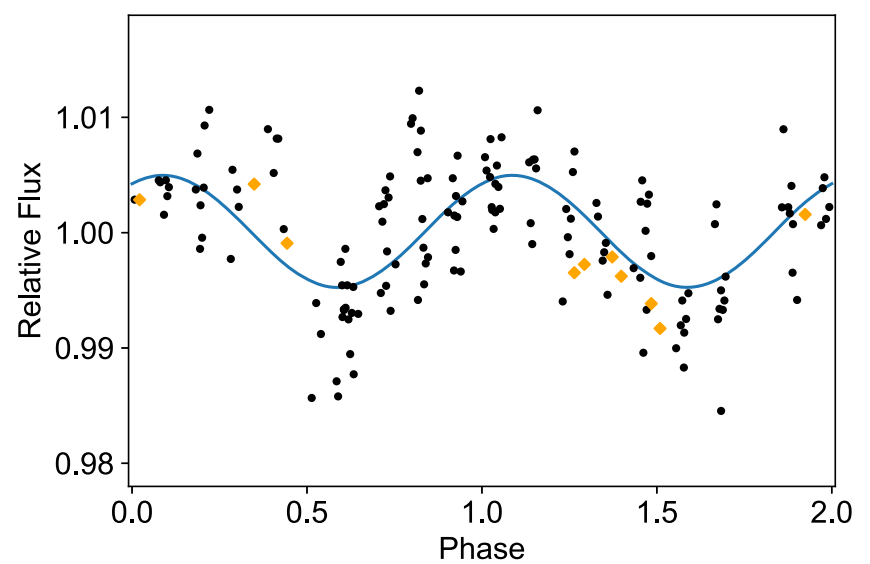

Figure 7. Ground-based light curves of EPIC 228732031 as a function of stellar rotational phase. The black circles are from AIT. The orange diamonds are from Swope. The blue line is a sinusoidal fit.

gives the results. In the circular model, the RV semiamplitude of planet $b$ is $6.77 \pm 1.50 \mathrm{~m} \mathrm{~s}^{-1}$, which translates into a planetary mass of $6.8 \pm 1.6 M_{\oplus}$. The mean density of the planet is $6.3_{-2.8}^{+3.1} \mathrm{~g} \mathrm{~cm}^{-3}$.

In the eccentric model, $K=6.64 \pm 1.55 \mathrm{~m} \mathrm{~s}^{-1}$, and the eccentricity is consistent with zero, $e<0.26$ (95\% confidence level). We compared the circular and eccentric models using the Bayesian information criterion, $B I C=-2 \times \log \left(\mathcal{L}_{\text {max }}\right)+$ $N \log (M)$, where $\mathcal{L}_{\max }$ is the maximum likelihood, $N$ is the number of parameters, and $M$ is the number of data points (Schwarz 1978; Liddle 2007). The circular model is favored by a $\triangle B I C=46.3$. For this reason, and because tidal dissipation is expected to circularize such a short-period orbit, in what follows we adopt the results from the circular model.

\subsection{Gaussian Process}

A Gaussian process is a model for a stochastic process in which a parametric form is adopted for the covariance matrix. Gaussian processes have been used to model the correlated noise in the RV data sets for several exoplanetary systems (e.g., Haywood et al. 2014; Grunblatt et al. 2015; López-Morales et al. 2016). Following Haywood et al. (2014), we chose a quasi-periodic kernel:

$$
\begin{aligned}
C_{i, j}= & h^{2} \exp \left[-\frac{\left(t_{i}-t_{j}\right)^{2}}{2 \tau^{2}}-\Gamma \sin ^{2} \frac{\pi\left(t_{i}-t_{j}\right)}{T}\right] \\
& +\left[\sigma_{i}^{2}+\sigma_{\mathrm{jit}}\left(t_{i}\right)^{2}\right] \delta_{i, j},
\end{aligned}
$$

where $C_{i, j}$ is an element of the covariance matrix, $\delta_{i, j}$ is the Kronecker delta function, $h$ is the covariance amplitude, $t_{i}$ is the time of $i$ th observation, $\tau$ is the correlation timescale, $\Gamma$ quantifies the relative importance between the squared exponential and periodic parts of the kernel, and $T$ is the period of the covariance. Note that $h, \tau, \Gamma$, and $T$ are the "hyperparameters" of the kernel. We chose this form for the kernel because the hyperparameters have simple physical interpretations in terms of stellar activity: $\tau$ and $\Gamma$ quantify the typical lifetime of active regions, and $T$ is closely related to the stellar rotation period. We also introduced a jitter term $\sigma_{\mathrm{jit}}$ specific to each instrument, to account for astrophysical and instrumental white noise.

The corresponding likelihood function has the following form:

$$
\log \mathcal{L}=-\frac{N}{2} \log 2 \pi-\frac{1}{2} \log |\boldsymbol{C}|-\frac{1}{2} \boldsymbol{r}^{\mathrm{T}} \boldsymbol{C}^{-1} \boldsymbol{r}
$$

where $\mathcal{L}$ is the likelihood, $N$ is the number of data points, $\boldsymbol{C}$ is the covariance matrix, and $\boldsymbol{r}$ is the residual vector (the observed $\mathrm{RV}$ minus the calculated value). The model includes the RV variation induced by the planet and a constant offset for each observatory. Based on the preceding results, we assumed the orbit to be circular. To summarize, the list of parameters is as follows: the jitter parameter and offset for each of the two spectrographs; the hyperparameters $h, \tau, \Gamma$, and $T$; and for each planet considered, its RV semiamplitude $K$, the orbital period $P_{\mathrm{orb}}$, and the time of conjunction $t_{\mathrm{c}}$. If nonzero eccentricity is allowed, two more parameters were added for each planet: $\sqrt{e} \cos \omega$ and $\sqrt{e} \sin \omega$. Again we imposed Gaussian priors on $P_{\text {orb }}$ and $t_{\mathrm{c}}$ for the planet $\mathrm{b}$ based on the fit to the transit light curve. We imposed Jeffreys priors on $h, K$, and the jitter parameters. We imposed uniform priors on $\gamma_{\mathrm{tab} 3}, \gamma_{\mathrm{tab} 4}, \sqrt{e} \cos \omega$ $([-1,1])$, and $\sqrt{e} \sin \omega([-1,1])$. The hyperparameters $\tau, \Gamma$, and 

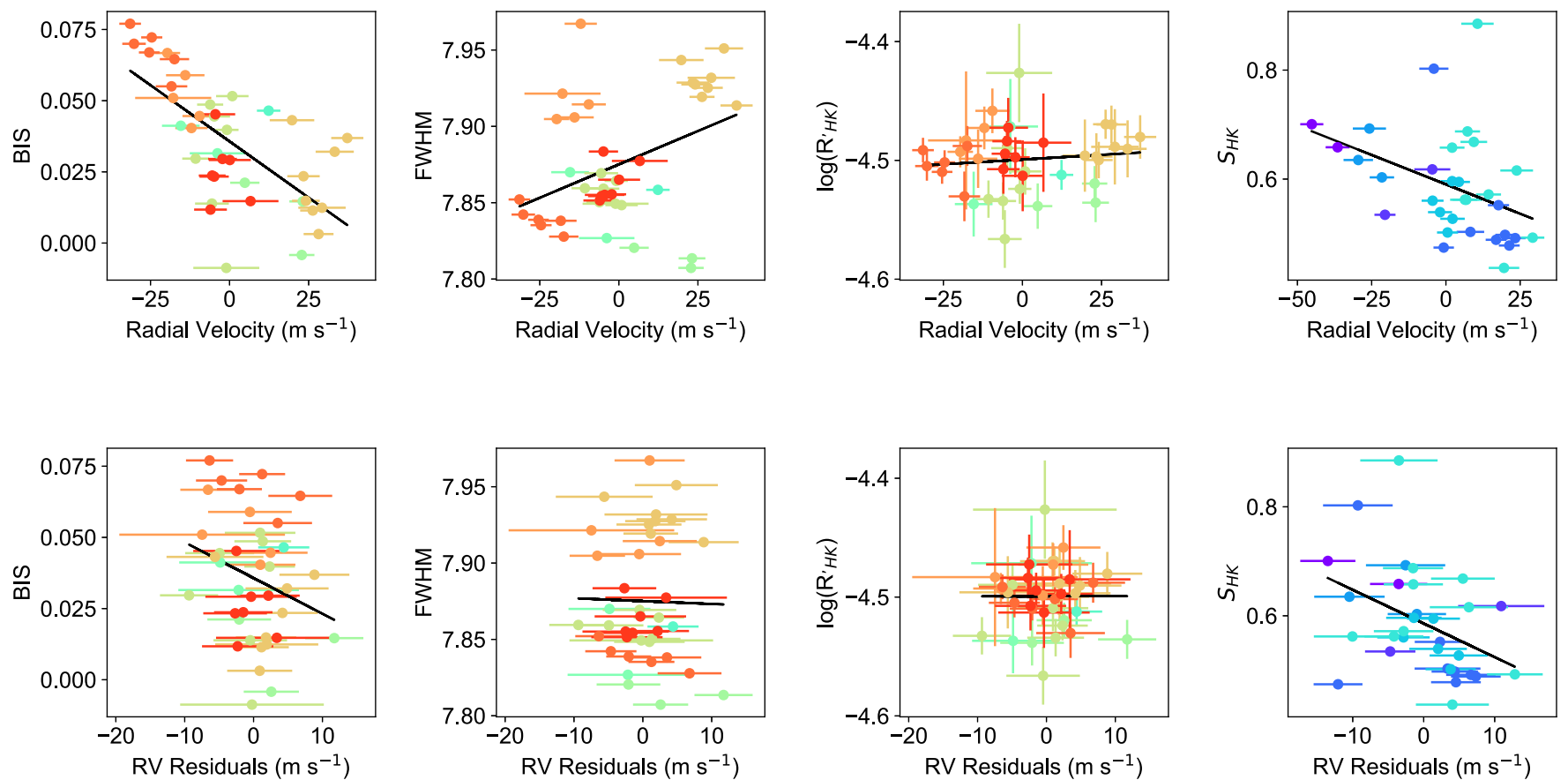

Figure 8. Top: measured RV plotted against various stellar activity indicators. Observations from different nights are plotted in different colors. The black line is the best-fit linear correlation between RV and the activity indicator. Among these, BIS, FWHM, and $S_{\mathrm{HK}}$ showed statistically significant correlation with the measured $\mathrm{RV}$, with Pearson $p$ values of $2.4 \times 10^{-6}, 0.014$, and 0.027 , respectively. Bottom: same, but using the residual RV after removing the Gaussian process regression model. This model largely succeeded in removing the correlations between RV and activity indicators. None of the activity indicators showed a statistically significant correlation with the measured RV.

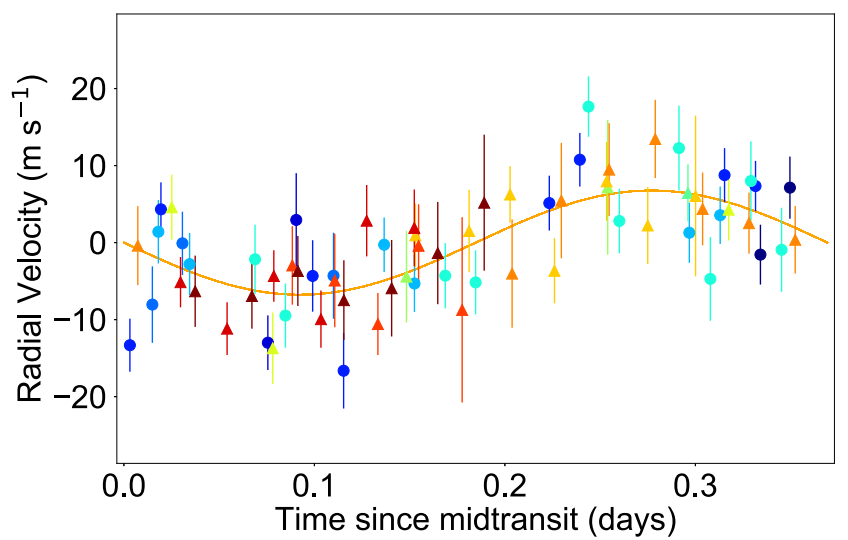

Figure 9. The best-fit model assuming a circular orbit using the floating chunk method. Each color shows the data from a single night. The circles are PFS data, and the triangles are HARPS-N data. The orange line is the best-fitting model.

$T$ were constrained through Gaussian process regression of the observed light curve, as described below.

\subsubsection{Photometric Constraints on the Hyperparameters}

The star's active regions produce apparent variations in both the RV and flux. Since the activity-induced flux variation and the radial velocity variation share the same physical origin, it is reasonable that they can be described by similar Gaussian processes (Aigrain et al. 2012). We used the $K 2$ and the ground-based photometry to constrain the hyperparameters, since the photometry has higher precision and better time sampling than the RV data.

When modeling the photometric data, we used the same form for the covariance matrix (Equation (2)) and the
Table 7

Planetary Parameters of EPIC 228732031b

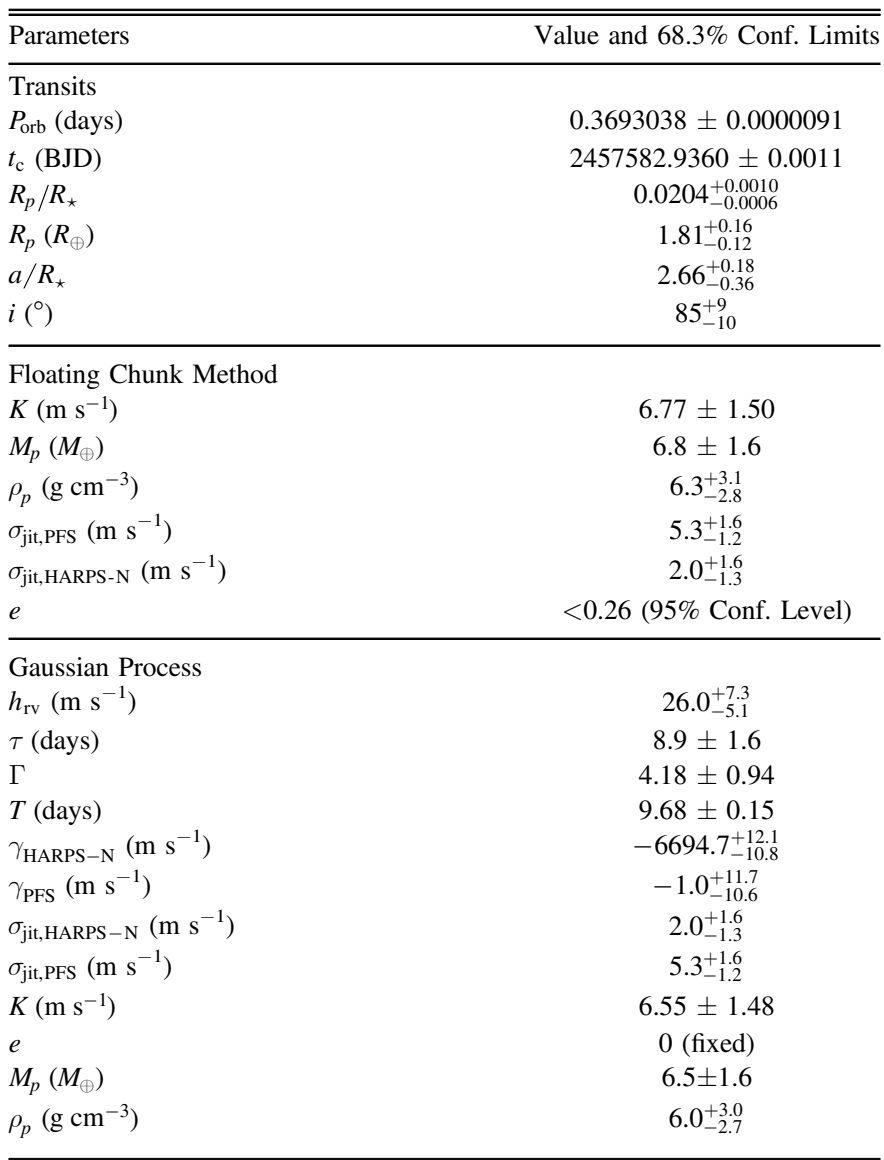




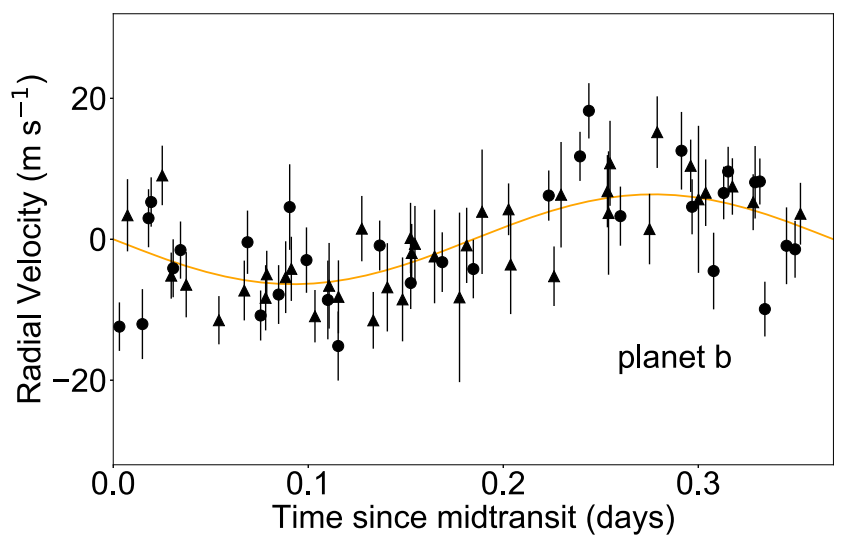

Figure 10. The best-fit circular-orbit model for planet b, using Gaussian process regression. The model for the correlated stellar noise (the blue dotted line in Figure 11) has been subtracted. The circles are PFS data, and the triangles are HARPS-N data.

likelihood function (Equation (3)). However, we replaced $h$ and $\sigma_{\text {jit }}$ with $h_{\text {phot }}$ and $\sigma_{\text {phot }}$ since the RV and photometric data have different units. The residual vector $\boldsymbol{r}$ in this case designates the measured flux minus a constant flux $f_{0}$. We also imposed a Gaussian prior on $T$ of $9.37 \pm 1.85$ days. We imposed Jeffreys priors on $h_{\text {phot, } K 2}, h_{\text {phot, AIT }}, h_{\text {phot, Swope }}, \sigma_{\text {phot, } K 2}, \sigma_{\text {phot, AIT }}$, $\sigma_{\text {phot, Swope }} \tau$, and $\Gamma$. We imposed uniform priors on $f_{0, K 2}$, $f_{0, \text { AIT }}$, and $f_{0 \text {, Swope }}$.

We found the best-fit solution using the Nelder-Mead algorithm implemented in the Python package scipy. Figures 1 and 6 show the best-fitting Gaussian process regression and its uncertainty range. To sample the posterior distributions, we used emcee, as described in Section 6.2. The posterior distributions are smooth and unimodal, leading to the following results for the hyperparameters: $\tau=9.5 \pm 1.0$ days, $\Gamma=3.32 \pm 0.58$, and $T=9.64 \pm 0.12$ days. These were used as priors in the Gaussian process analysis of the RV data.

\subsection{Mass of Planet $b$}

With the constraints on the hyperparameters obtained from the previous section, we analyzed the measured RV with Gaussian process regression. We found the best-fit solution using the Nelder-Mead algorithm implemented in the Python package scipy. Allowing for a nonzero eccentricity did not lead to an improvement in the BIC, so we assumed the orbit to be circular for the subsequent analysis. We sampled the parameter posterior distribution, again using emcee, giving smooth and unimodal distributions. Table 7 reports the results, based on the $16 \%, 50 \%$, and $84 \%$ levels of the distributions. The RV semiamplitude for planet $\mathrm{b}$ is $6.55 \pm 1.48 \mathrm{~m} \mathrm{~s}^{-1}$, which is consistent with that obtained with the floating chunk method. This translates into a planetary mass of $6.5 \pm 1.6 M_{\oplus}$ and a mean density of $6.0_{-2.7}^{+3.0} \mathrm{~g} \mathrm{~cm}^{-3}$. Figure 10 shows the signal of planet $b$ after removing the correlated stellar noise.

Figure 11 shows the measured RV variation of EPIC 228732031 and the Gaussian process regression. The correlated noise component dominates the model for the observed radial velocity variation. The amplitude of the correlated noise is $h_{\mathrm{rv}}=26.0_{-5.1}^{+7.3} \mathrm{~m} \mathrm{~s}^{-1}$. This is consistent with the level of correlated noise we expected from stellar activity. Based on the observed amplitude of photometric modulation and the projected stellar rotational velocity, we expected

$$
h_{\mathrm{rv}} \approx v \sin i_{\star} \times h_{\mathrm{phot}}=4.4 \mathrm{~km} \mathrm{~s}^{-1} \times 0.005 \approx 22 \mathrm{~m} \mathrm{~s}^{-1} \text {. }
$$

The Gaussian process regression successfully removed most of the correlated noise, as well as the correlations between the measured RV and the activity indicators. This is shown in the lower panel of Figure 8. The clustering of nightly observations seen in the original data set (upper panel) was significantly reduced. Pearson correlation tests showed that none of the activity indicators correlate significantly with the radial velocity residuals.

\subsubsection{Planet $c$ ?}

Many of the detected USP planets have planetary companions (See Table 8). Although the $K 2$ light curve did not reveal another transiting planet (Section 6.1), there could be the signal of a nontransiting planet lurking in our radial velocity data set. We addressed this question by scrutinizing the Lomb-Scargle periodograms of the light curves, radial velocities, and various activity indicators (See Figure 12).

The stellar rotation period of 9.4 days showed up clearly in both the $K 2$ and ground-based light curves. However, the same periodicity is not significant in the periodogram of the measured radial velocities. The strongest peak in the RV periodogram occurs at $3.0_{-0.06}^{+0.19}$ days with a false-alarm probability $<0.001$, shown with a green dotted line in Figure 12. This raises the question of whether this 3-day signal is due to a nontransiting planet or stellar activity. Based on the following reasons, we argue that stellar activity is the more likely possibility.

The signal at 3.0 $0_{-0.06}^{+0.19}$ days is suspiciously close to the second harmonic $P_{\text {rot }} / 3$ of the stellar rotation period (9.4 days). Previous simulations by Vanderburg et al. (2016) showed that the radial velocity variations induced by stellar activity often have a dominant periodicity at the first or second harmonics of the stellar rotation period (see their Figure 6). Aigrain et al. (2012) presented the $F F^{\prime}$ method as a simple way to predict the radial velocity variations induced by stellar activity using precise and well-sampled light curves. Using the prescription provided by Aigrain et al. (2012) and the $K 2$ light curve, we estimated the activity-induced radial velocity variation of EPIC 228732031. As noted at the beginning of Section 7, the activity-induced radial velocity variation has both rotational and convective components, which are represented by Equations (10) and (12) of Aigrain et al. (2012). For EPIC 228732031, the Lomb-Scargle periodograms of both the rotational and convective components showed a strong periodicity at $P_{\text {rot }} / 3$ (see Figure 12). This suggests that the $3.0_{-0.06}^{+0.19}$ day periodicity in the measured $\mathrm{RV}$ is attributable to activity-induced RV variation rather than a nontransiting planet.

\section{Discussion}

\subsection{Composition of EPIC $228732031 b$}

To investigate the constraints on the composition of EPIC 228732031b, we appeal to the theoretical models of the interiors of super-Earths by Zeng et al. (2016). We initially considered a differentiated three-component model consisting of water, iron, and rock (magnesium silicate). We found 


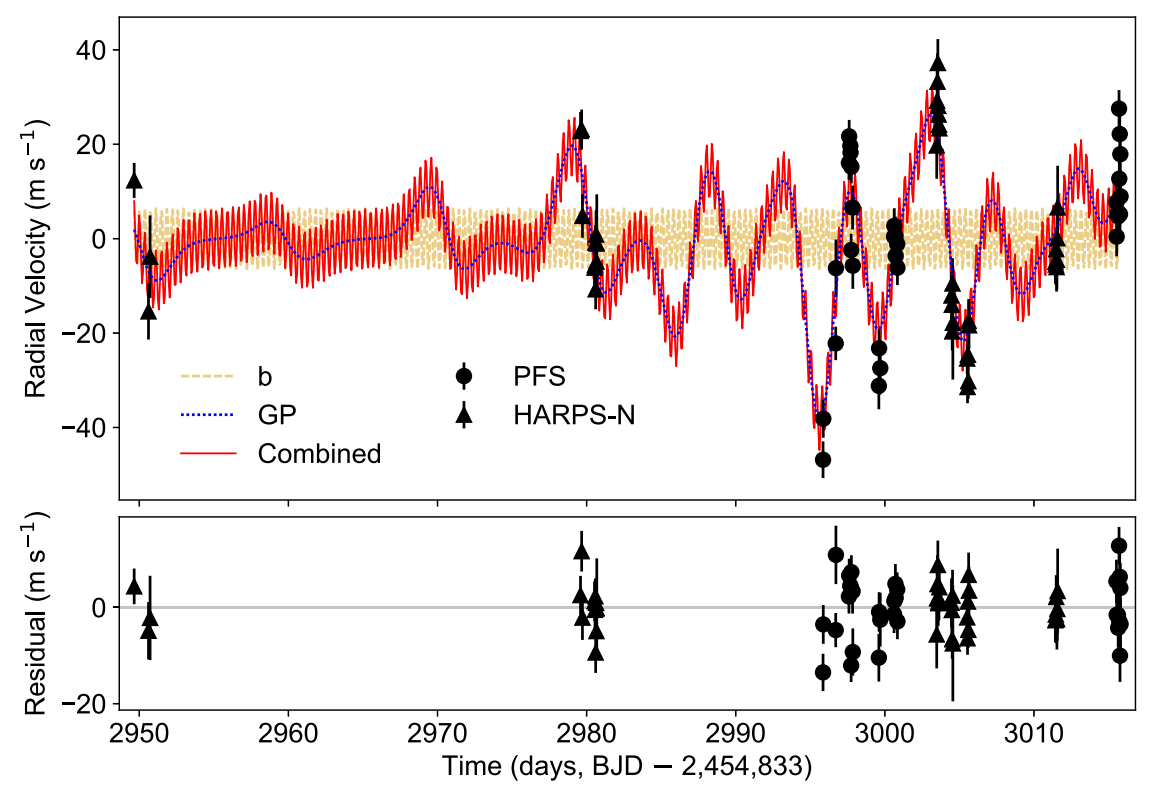

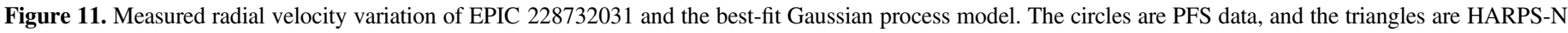

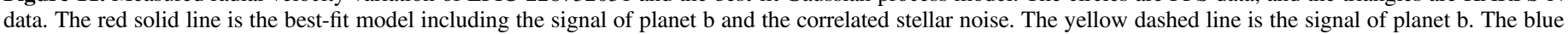
dotted line is the Gaussian process.

the constraints on composition to be quite weak. For EPIC 228732031b, the $1 \sigma$ confidence interval encompasses most of the iron/water/rock ternary diagram (see Figure 13).

We then considered two more restricted models. In the first model, the planet is a mixture of rock and iron only, without the water component. For EPIC 228732031b, the iron fraction can be $0 \%-44 \%$ and still satisfy the $1 \sigma$ constraint on the planetary mass and radius.

The second model retains all three components-rock, iron, and water-but requires the iron/rock ratio to be $3 / 7$, similar to the Earth. Thus in this model we determine the allowed range for the water component. For EPIC 228732031b, the allowed range for the water mass fraction is $0 \%-59 \%$.

\subsection{Composition of the Sample of USP Planets}

As we saw in the preceding section, the measurement of mass and radius alone does not place strong constraints on the composition of an individual planet. In the super-Earth regime (1-2 $R_{\oplus}$ or $1-10 M_{\oplus}$ ), there are many plausible compositions, which are difficult to distinguish based only on the mass and radius. To pin down the composition of any individual system, it will be necessary to increase the measurement precision substantially or obtain additional information, such as measurements of the atmospheric composition. With eight members, though, the sample of USP planets has now grown to the point at which trends in composition with size, or other parameters, might start to become apparent.

Table 8 summarizes the properties of all the known USP planets for which both the planetary mass and radius have been measured. The table has been arranged in order of increasing planetary radius. Figure 14 displays their masses and radii, along with representative theoretical mass-radius relationships from Zeng et al. (2016). We did not include KOI-1843.03 (Rappaport et al. 2013) and EPIC 228813918b (Smith et al. 2017) in this diagram since their masses have not been measured, although in both cases a lower limit on the density can be obtained by assuming the planets are outside of the
Roche limit. The data points are color-coded according to the level of irradiation by the star. One might have expected the more strongly irradiated planets to have a higher density, as a consequence of photoevaporation. However, we do not observe any correlation between planetary mean density and level of irradiation. This may be because all of the USP planets are so strongly irradiated that photoevaporation has gone to completion in all cases. Lundkvist et al. (2016) argued for a threshold of $650 F_{\oplus}$ (where $F_{\oplus}$ is the insolation level received by Earth) as the value above which close-in sub-Neptunes have undergone photoevaporation. All of the USP planets in Table 8 have much higher levels of irradiation than this threshold. Therefore, it is possible that all these planets have been entirely stripped of any preexisting hydrogen/helium atmospheres, and additional increases in irradiation would not affect the planetary mass or radius. Ballard et al. (2014) also found no correlation between irradiation and mean density within a sample of planets with measured masses, radii smaller than $2.2 R_{\oplus}$, and orbital periods $\lesssim 10$ days.

In the mass-radius diagram, the eight USP planets cluster between the theoretical relations for pure rock $\left(100 \% \mathrm{MgSiO}_{3}\right)$ and an Earth-like composition $\left(30 \% \mathrm{Fe}\right.$ and $\left.70 \% \mathrm{MgSiO}_{3}\right)$. Earlier work by Dressing et al. (2015) pointed out that the bestcharacterized planets with masses smaller than $6 M_{\oplus}$ are consistent with a composition of $17 \% \mathrm{Fe}$ and $83 \% \mathrm{MgSiO}_{3}$. Their sample of planets consisted of Kepler-78b, Kepler-10b, CoRoT-7b, Kepler-93b, and Kepler-36b. Of these, the first three are USP planets; the latter two have orbital periods of 4.7 and 13.8 days. Dressing et al. (2015) also claimed that planets heavier than $6 M_{\oplus}$ usually have a gaseous $\mathrm{H} / \mathrm{He}$ envelope or a significant contribution of low-density volatiles-presumably water-to the planet's total mass. Similarly, Rogers (2015) sought evidence for a critical planet radius separating rocky planets and those with gaseous or water envelopes. She found that for planets with orbital periods shorter than 50 days, those that are smaller than $1.6 R_{\oplus}$ are predominantly rocky, whereas larger planets usually have gaseous or volatile-enhanced envelopes. 
Table 8

Ultra-short-period Planets with Mass Measurements

\begin{tabular}{|c|c|c|c|c|c|c|c|c|c|c|c|c|}
\hline & $\begin{array}{l}T_{\text {eff }} \\
(\mathrm{K})\end{array}$ & $\begin{array}{c}{[\mathrm{Fe} / \mathrm{H}]} \\
(\mathrm{dex})\end{array}$ & $\begin{array}{c}M_{\star} \\
\left(M_{\odot}\right)\end{array}$ & $\begin{array}{c}R_{\star} \\
\left(R_{\odot}\right)\end{array}$ & $\begin{array}{c}P \\
\text { (days) }\end{array}$ & $\begin{array}{c}R_{p} \\
\left(R_{\oplus}\right)\end{array}$ & $\begin{array}{c}M_{p} \\
\left(M_{\oplus}\right)\end{array}$ & $\begin{array}{c}\rho_{p} \\
\left(\mathrm{~g} \mathrm{~cm}^{-3}\right)\end{array}$ & $N_{\mathrm{pl}}$ & $\mathrm{Fe}-\mathrm{MgSiO}_{3}{ }^{\mathrm{a}}$ & $\mathrm{H}_{2} \mathrm{O}^{\mathrm{b}}$ & Reference \\
\hline Kepler-78b & $5089 \pm 50$ & $-0.14 \pm 0.08$ & $0.83 \pm 0.05$ & $0.74 \pm 0.05$ & 0.36 & $1.20 \pm 0.09$ & $1.87_{-0.26}^{+0.27}$ & $6.0_{-1.4}^{+1.9}$ & 1 & $36 \%-64 \%$ & $<57 \%$ & Ho13, Gr15 \\
\hline Kepler-10b & $5627 \pm 44$ & $-0.09 \pm 0.04$ & $0.913 \pm 0.022$ & $1.065 \pm 0.009$ & 0.84 & $1.47_{-0.02}^{+0.03}$ & $3.72 \pm 0.42$ & $6.46 \pm 0.73$ & 2 & $21 \%-79 \%$ & $2_{-2}^{+10} \%$ & Ba11, We16 \\
\hline CoRot-7b & $5250 \pm 60$ & $0.12 \pm 0.06$ & $0.91 \pm 0.03$ & $0.82 \pm 0.04$ & 0.85 & $1.585 \pm 0.064$ & $4.73 \pm 0.95$ & $6.61 \pm 1.33$ & 2 & $15 \%-85 \%$ & $3_{-3}^{+23} \%$ & Br10, Ha14 \\
\hline K2-106b & $5470 \pm 30$ & $0.025 \pm 0.020$ & $0.945 \pm 0.063$ & $0.869 \pm 0.088$ & 0.57 & $1.52 \pm 0.16$ & $8.36_{-0.94}^{+0.96}$ & $13.1_{-3.6}^{+5.4}$ & 2 & $80 \%-20 \%$ & $<20 \%$ & Gu17 \\
\hline K2-106b & $5496 \pm 46$ & $0.06 \pm 0.03$ & $0.95 \pm 0.05$ & $0.92 \pm 0.03$ & 0.57 & $1.82_{-0.14}^{+0.2}$ & $9.0 \pm 1.6$ & $8.57_{-2.80}^{+4.64}$ & 2 & $26 \%-74 \%$ & $2_{-2}^{+27} \%$ & Si17 \\
\hline HD $3167 b$ & $5286 \pm 40$ & $0.02 \pm 0.03$ & $0.877 \pm 0.024$ & $0.835 \pm 0.026$ & 0.96 & $1.575 \pm 0.054$ & $5.69 \pm 0.44$ & $8.00_{-0.98}^{+1.10}$ & $2-4$ & $37 \%-63 \%$ & $<10 \%$ & Ga17 \\
\hline HD $3167 b$ & $5261 \pm 60$ & $0.04 \pm 0.05$ & $0.86 \pm 0.03$ & $0.86 \pm 0.04$ & 0.96 & $1.70_{-0.15}^{+0.18}$ & $5.02 \pm 0.38$ & $5.60_{-1.43}^{+2.15}$ & 3 & At least $6 \% \mathrm{H}_{2} \mathrm{O}$ & $15_{-15}^{+35} \%$ & Ch17 \\
\hline EPIC $228732031 b$ & $5200 \pm 100$ & $0.00 \pm 0.08$ & $0.84 \pm 0.03$ & $0.81 \pm 0.03$ & 0.37 & $1.81_{-0.12}^{+0.16}$ & $6.5 \pm 1.6$ & $6.0_{-2.7}^{+3.0}$ & 1 & At least $4 \% \mathrm{H}_{2} \mathrm{O}$ & $15_{-15}^{+44} \%$ & This Work \\
\hline WASP-47e & $5576 \pm 68$ & $0.36 \pm 0.05$ & $1.04 \pm 0.031$ & $1.137 \pm 0.013$ & 0.79 & $1.81 \pm 0.027$ & $6.83 \pm 0.66$ & $6.35 \pm 0.78$ & 4 & At least $2 \% \mathrm{H}_{2} \mathrm{O}$ & $12_{-8}^{+10} \%$ & Be15, Va17 \\
\hline $55 \mathrm{Cnc}$ e & $5196 \pm 24$ & $0.31 \pm 0.04$ & $0.905 \pm 0.015$ & $0.943 \pm 0.010$ & 0.74 & $1.92 \pm 0.08$ & $8.08 \pm 0.31$ & $6.4_{-0.7}^{+0.8}$ & 5 & At least $4 \% \mathrm{H}_{2} \mathrm{O}$ & $16_{-10}^{+35} \%$ & Va05, Br11, De16 \\
\hline
\end{tabular}

Notes.

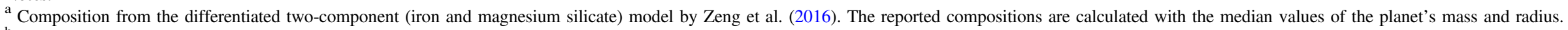

${ }^{\mathrm{b}}$ Water mass fraction assuming a water envelope on top of an Earth-like core of $30 \% \mathrm{Fe}-70 \% \mathrm{MgSiO}_{3}$. The upper limits are quoted at the $95 \%$ confidence level.

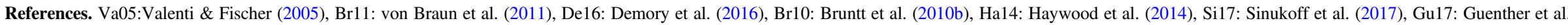

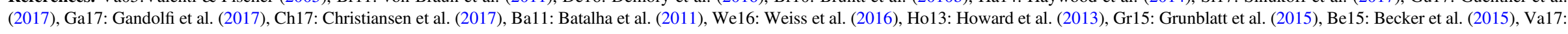
Vanderburg et al. (2017). 

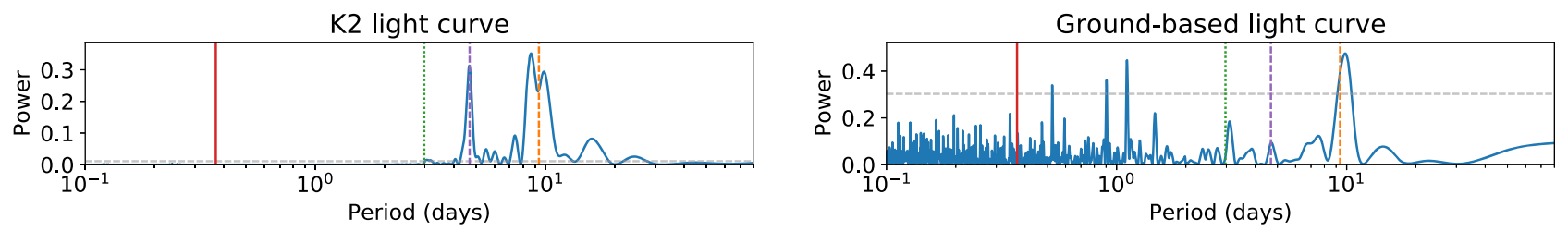

RV
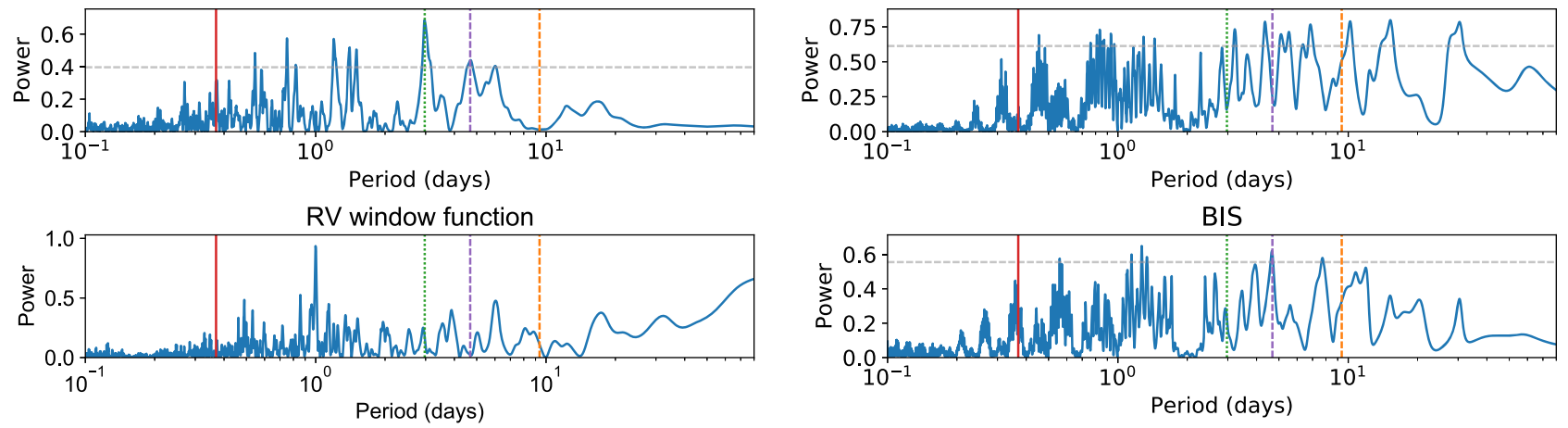

FF' rotational
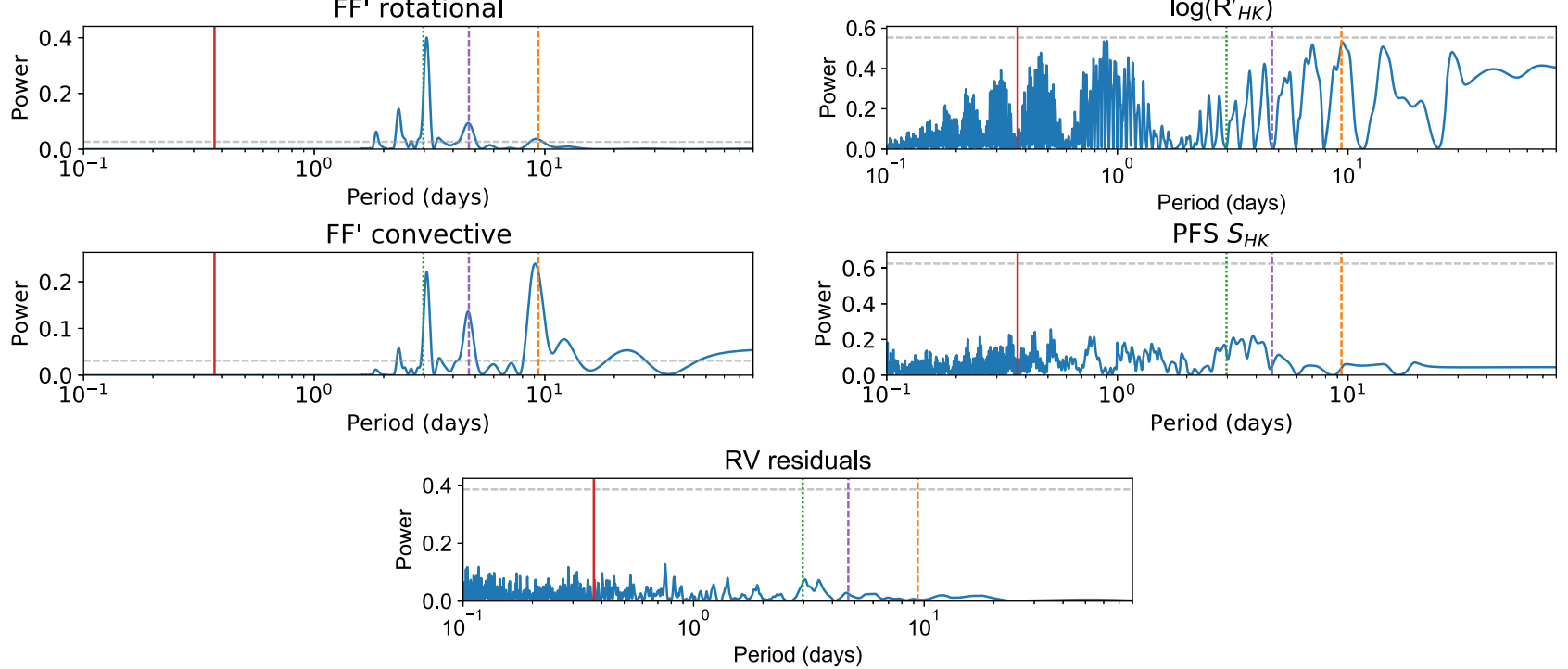

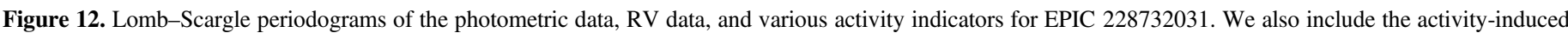

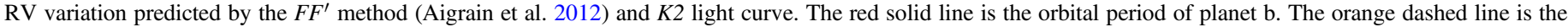

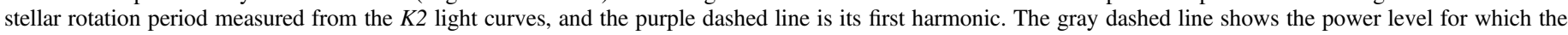

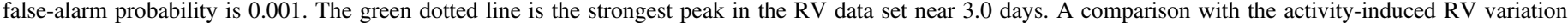
predicted by the $F F^{\prime}$ method (Aigrain et al. 2012) shows that 3.0-day periodicity is likely due to stellar activity.

Could the same hold true for the USP planets? That is, could the larger and more massive USP planets hold on to a substantial water envelope, despite the extreme irradiation they experience? Lopez (2016) investigated this question from a theoretical point of view and argued that such an envelope could withstand photoevaporation. He found that USP planets are able to retain water envelopes even at irradiation levels of about $2800 F_{\oplus}$. If this is true, then we would expect the USP planets larger than $1.6 R \oplus$ to be more massive than $6 M_{\oplus}$ and to have densities low enough to be compatible with a water envelope.

Of the eight USP planets for which mass and radius have both been measured, five are larger than $1.6 R_{\oplus}: 55 \mathrm{Cnc}$ e, WASP-47e, EPIC 228732031b, HD 3167b, and K2-106b. Interestingly, they also have masses heavier than $6 M_{\oplus}$. The first three of these (55 Cnc e, WASP-47e, EPIC 228732031b) have a low mean density compatible with a water envelope. Applying the second model described above, in which a "terrestrial" core (30\% iron, $70 \%$ rock) is supplemented with a water envelope, we find that these four planets all have water mass fractions $>10 \%$ at the best-fit values of their mass and radius. For K2-106b and HD 3167b, two different groups have reported different values for the masses and radii, leading to different conclusions about their composition. For K2-106b, Sinukoff et al. (2017) reported a planetary mass and radius of $9.0 \pm 1.6 M_{\oplus}$ and $1.82_{-0.14}^{+0.20} R_{\oplus}$, suggesting a rocky composition. Guenther et al. (2017) reported a planetary mass and radius of $8.36_{-0.94}^{0.96} M_{\oplus}$ and $1.52 \pm 0.16 R_{\oplus}$, which pointed to an iron-rich composition. In both cases, the mean density of K2-106b seems to defy the simple interpretation that planets more massive than $6 M_{\oplus}$ have $\mathrm{H} / \mathrm{He}$ or water envelopes. For HD 3167b, Christiansen et al. (2017) reported a planetary mass and radius of $5.02 \pm 0.38 M_{\oplus}$ and $1.70_{-0.15}^{+0.18} R_{\oplus}$ (consistent with water mass fractions of $>10 \%$ ), whereas Gandolfi et al. (2017) reported $5.69 \pm 0.44 M_{\oplus}$ and $1.575 \pm 0.054 R_{\oplus}$, suggesting a predominantly rocky composition. Given the different results 


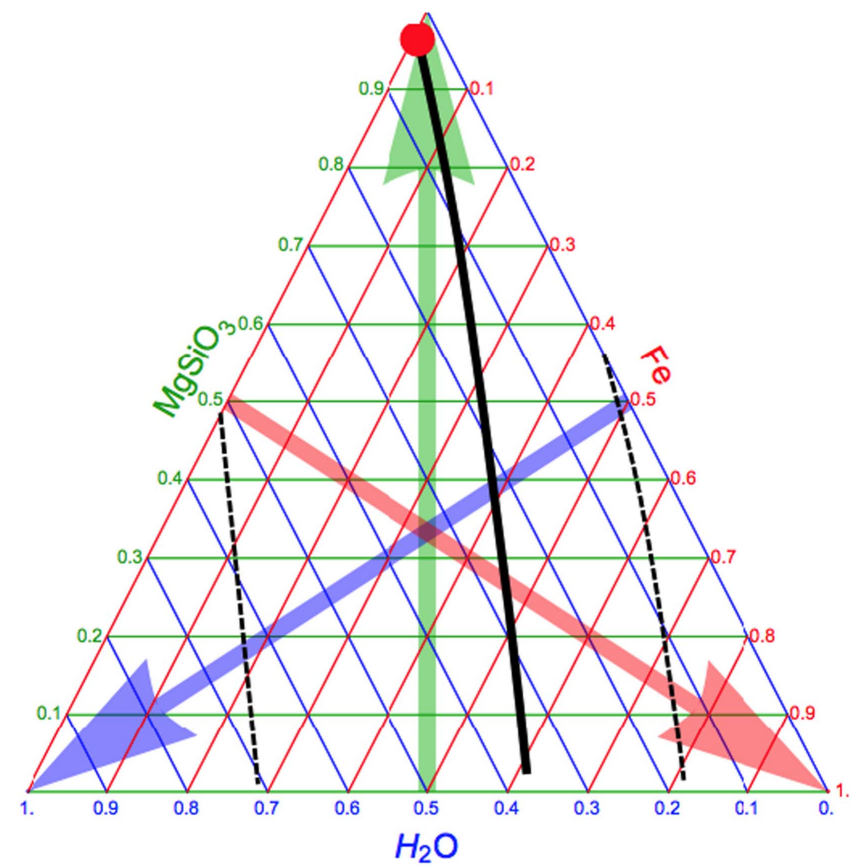

Figure 13. The $\mathrm{H}_{2} \mathrm{O}-\mathrm{MgSiO}_{3}-\mathrm{Fe}$ ternary diagram for the interior composition of EPIC 228732031b (Zeng et al. 2016). The solid line is the locus corresponding to the best-fit values of the planetary radius and mass. The dashed lines show the $1 \sigma$ confidence interval.

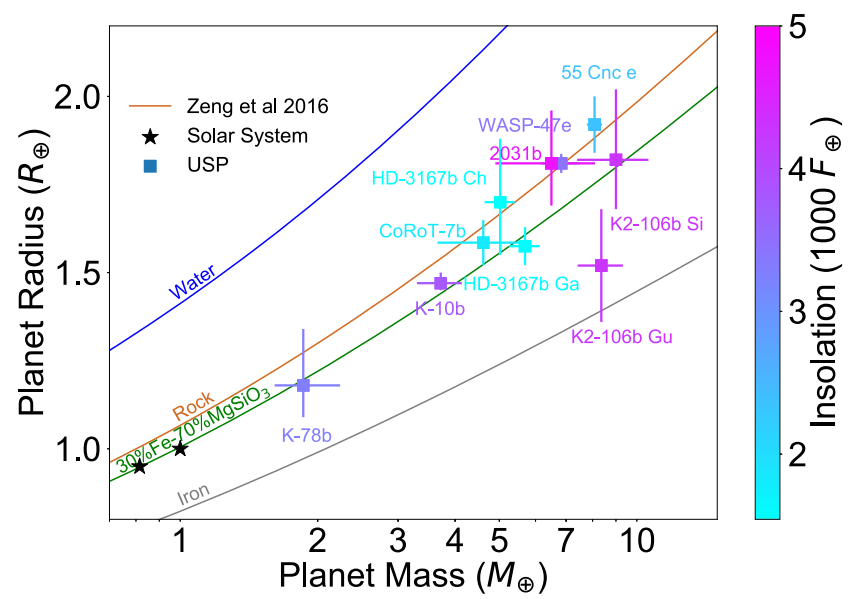

Figure 14. Mass and radius of USPs along with theoretical mass-radius curves from Zeng et al. (2016). The stars are solar system planets. Color indicates the level of insolation in units of $F_{\oplus}$, the insolation level received by Earth. For K2$106 \mathrm{~b}$, we plot the results from both Guenther et al. (2017) and Sinukoff et al. (2017). For HD 3167b, we plot the results from both Christiansen et al. (2017) and Gandolfi et al. (2017).

reported by the various groups and the fact that the radii of $\mathrm{K} 2$ 106b and HD 3167b lie so close to the transition radius of $1.6 R_{\oplus}$ identified by Rogers (2015), the interpretation of these two planets is still unclear. More data, or at least a joint analysis of all the data collected by both groups, would probably help to clarify the situation.

A substantial water envelope for $55 \mathrm{Cnc}$ e, WASP-47e, and EPIC 228732031b would have implications for the formation of those planets. Theories in which these planets form in situ (i.e., near their current orbits) would have difficulty explaining the presence of icy material and the runaway accretion of the gas giant within the snow line. Thus a massive water envelope would seem to imply that the planet formed beyond the snow line, unless there were some efficient mechanism for delivering water to the inner portion of the planetary system. To move a planet from beyond the snow line to a very tight orbit, theorists have invoked disk migration or high-eccentricity migration. The observed architectures of the USP planetary systems, described below, seem to be dynamically cold, and therefore more compatible with disk migration than high-eccentricity migration.

It is interesting to note that $55 \mathrm{Cnc}$ e and WASP-47b, the most extensively studied planets in the USP planet sample, show some striking similarities. Both systems appear to be dynamically cold. The inner three planets of WASP-47 are all transiting, indicating low mutual inclinations (Becker et al. 2015), and Sanchis-Ojeda et al. (2015) ruled out a very large stellar obliquity $\left(\lambda=04^{\circ} \pm 24^{\circ}\right)$. Dynamical analysis of the $55 \mathrm{Cnc}$ system suggests that the system is nearly coplanar, which helps maintain long-term stability (Nelson et al. 2014). The dynamically cold configuration of both $55 \mathrm{Cnc}$ and WASP-47 seems to disfavor any formation scenario involving high-eccentricity migration, which would likely produce a dynamically hot final state. These two systems also share a few other intriguing and potentially relevant properties. Among the USP planet sample, they have the largest number of detected planets (five and four) and are the only systems in which gas giants are also known to be present (in 14.6-day and 4.2-day orbits). They also have the most metal-rich stars, with $[\mathrm{Fe} / \mathrm{H}]$ of $0.31 \pm 0.04$ and $0.36 \pm 0.05$, as compared to the mean $[\mathrm{Fe} / \mathrm{H}]$ of $0.0018 \pm 0.0051$ for the $62 \mathrm{USP}$ systems studied by Winn et al. (2017). The unusually high metallicity of these two systems might help to explain the large number of detected planets and the presence of giant planets in these systems. As argued by Dawson et al. (2016), a metal-rich protoplanetary disk likely has a higher solid surface density. The higher solid surface density facilitates the formation and assembly of planet embryos. As a result, we might expect a metal-rich disk to spawn more planets and to facilitate the growth of solid cores past the critical core mass needed for giant planet formation.

\subsection{Other Properties of the USP Planets}

Sanchis-Ojeda et al. (2014) pointed out that USP planets commonly have wider-orbiting planetary companions. About $10 \%$ of their sample of USP planets had longer-period transiting companions. After taking into account the decline in transit probability with orbital period, they inferred that nearly all of the planets in their sample are likely to have longer-period companions, most of which are not transiting. Of the eight USP planets in Table 8, six of them have confirmed wider-orbiting companions. The only known exceptions are Kepler-78 and EPIC 228732031, which are probably also the least explored for additional planets. A problem in both cases is the high level of stellar activity: the light curves display clear rotational modulations with periods of 12.5 days and 9.4 days, and the existing RV data show evidence for activity-related noise that hinders the detection of additional planets (Howard et al. 2013; Sanchis-Ojeda et al. 2013).

In summary, observations of systems with USP planets have revealed the following properties: (1) They tend to have a high multiplicity of planets. (2) The high multiplicity and the measured properties of a few USP systems suggest that they have a dynamically cold architecture characterized by low mutual inclinations and (possibly) a low stellar obliquity. (3) The metallicity distribution of USP planet hosts is inconsistent 
with the metallicity distribution of hot Jupiter hosts and indistinguishable from that of the close-in sub-Neptunes (Winn et al. 2017). (4) At least three of the five USP planets known to be larger than $1.6 R_{\oplus}$ are also heavier than $6 M_{\oplus}$ and have densities low enough to be compatible with a water envelope. Taken together, these observations suggest to us that the USP planets are a subset of the general population of sub-Neptune planets, most of which have lost their atmospheres entirely to photoevaporation, except for the largest few that have retained a substantial water envelope.

This work was carried out as part of the KESPRINT consortium. This paper includes data gathered with the $6.5 \mathrm{~m}$ Magellan Telescopes located at Las Campanas Observatory, Chile. This work is based on observations made with the Italian Telescopio Nazionale Galileo (TNG) operated on the island of La Palma by the Fundación Galileo Galilei of the INAF (Istituto Nazionale di Astrofisica) at the Spanish Observatorio del Roque de los Muchachos of the Instituto de Astrofísica de Canarias, as part of the observing programs A34TAC_10 and A34TAC_44. We are very grateful to the TNG staff members for their precious support during the observations. Some of the data presented in this work were obtained at the WIYN Observatory from telescope time allocated to NN-EXPLORE through the scientific partnership of the National Aeronautics and Space Administration, the National Science Foundation, and the National Optical Astronomy Observatory. This work was supported by a NASA WIYN PI Data Award, administered by the NASA Exoplanet Science Institute. NESSI was funded by the NASA Exoplanet Exploration Program and the NASA Ames Research Center. NESSI was built at the Ames Research Center by Steve B. Howell, Nic Scott, Elliott P. Horch, and Emmett Quigley. The authors wish to recognize and acknowledge the very significant cultural role and reverence that the summit of Maunakea has always had within the indigenous Hawaiian community. We are most fortunate to have the opportunity to conduct observations from this mountain. The authors are honored to be permitted to conduct observations on Iolkam Duag (Kitt Peak), a mountain within the Tohono O'odham Nation with particular significance to the Tohono O'odham people. D.G. gratefully acknowledges the financial support of the Programma Giovani Ricercatori-Rita Levi Montalcini-Rientro dei Cervelli (2012) awarded by the Italian Ministry of Education, Universities and Research (MIUR).

Facilities: Kepler (K2), Fairborn Observatory/AIT, WIYN/ NESSI, LCO/Swope, Magellan/PFS, TNG/HARPS-N.

Software: ATLAS12 (Kurucz 2013), Python, Batman (Kreidberg 2015), emcee (Foreman-Mackey et al. 2013), iSpec (Blanco-Cuaresma et al. 2014), Isochrones (Morton 2015), lmfit (Newville et al. 2014), MARCS (Gustafsson et al. 2008), MOOG (Sneden 1973), MultiNest (Feroz et al. 2009), NEXTGEN (Hauschildt et al. 1999), scipy (Jones et al. 2001), SME (v5.22 Valenti \& Piskunov 1996; Valenti \& Fischer 2005; Piskunov \& Valenti 2017), SpecMatch-emp 6 (Yee et al. 2017), SPECTRUM3 (V2.76 Gray \& Corbally 1994), VALD3 (Piskunov et al. 1995; Kupka \& Ryabchikova 1999).

\section{ORCID iDs}

Fei Dai (i) https://orcid.org/0000-0002-8958-0683

Joshua N. Winn (10) https://orcid.org/0000-0002-4265-047X Davide Gandolfi (i) https://orcid.org/0000-0001-8627-9628
Jennifer Burt (1) https://orcid.org/0000-0002-0040-6815 William D. Cochran (1) https://orcid.org/0000-00019662-3496

Michael Endl (1) https://orcid.org/0000-0002-7714-6310 Malcolm Fridlund (1) https://orcid.org/0000-0003-2180-9936 Marshall C. Johnson (i) https://orcid.org/0000-00025099-8185

Andrew Vanderburg (10 https://orcid.org/0000-00017246-5438

Pamela Arriagada (1) https://orcid.org/0000-0002-3578-551X R. P. Butler (i) https://orcid.org/0000-0003-1305-3761 Juan Cabrera 누 https://orcid.org/0000-0001-6653-5487 Jeffrey D. Crane (i) https://orcid.org/0000-0002-5226-787X Felice Cusano (1) https://orcid.org/0000-0003-2910-6565 Hans Deeg (1) https://orcid.org/0000-0003-0047-4241 Mark E. Everett (10) https://orcid.org/0000-0002-0885-7215 Akihiko Fukui (ํ) https://orcid.org/0000-0002-4909-5763 Gregory W. Henry (1) https://orcid.org/0000-0003-4155-8513 Steve B. Howell (1) https://orcid.org/0000-0002-2532-2853 Norio Narita (1) https://orcid.org/0000-0001-8511-2981 Grzegorz Nowak (1) https://orcid.org/0000-0002-7031-7754 Robert A. Wittenmyer (1) https://orcid.org/0000-00019957-9304

\section{References}

Aigrain, S., Pont, F., \& Zucker, S. 2012, MNRAS, 419, 3147 Axer, M., Fuhrmann, K., \& Gehren, T. 1994, A\&A, 291, 895 Ballard, S., Chaplin, W. J., Charbonneau, D., et al. 2014, ApJ, 790, 12 Batalha, N. M., Borucki, W. J., Bryson, S. T., et al. 2011, ApJ, 729, 27 Becker, J. C., Vanderburg, A., Adams, F. C., Rappaport, S. A., \& Schwengeler, H. M. 2015, ApJL, 812, L18

Blanco-Cuaresma, S., Soubiran, C., Heiter, U., \& Jofré, P. 2014, A\&A, 569, A111

Bruntt, H., Bedding, T. R., Quirion, P.-O., et al. 2010a, MNRAS, 405, 1907 Bruntt, H., Deleuil, M., Fridlund, M., et al. 2010b, A\&A, 519, A51 Butler, R. P., Marcy, G. W., Williams, E., et al. 1996, PASP, 108, 500

Cardelli, J. A., Clayton, G. C., \& Mathis, J. S. 1989, ApJ, 345, 245

Castelli, F., \& Kurucz, R. L. 2004, arXiv:astro-ph/0405087

Christiansen, J. L., Vanderburg, A., Burt, J., et al. 2017, AJ, in press, arXiv: 1706.01892

Cosentino, R., Lovis, C., Pepe, F., et al. 2012, Proc. SPIE, 8446, 84461V

Crane, J. D., Shectman, S. A., Butler, R. P., et al. 2010, Proc. SPIE, 7735, 53

Dawson, R. I., Lee, E. J., \& Chiang, E. 2016, ApJ, 822, 54

Demory, B.-O., Gillon, M., Madhusudhan, N., \& Queloz, D. 2016, MNRAS, 455, 2018

Dotter, A., Chaboyer, B., Jevremović, D., et al. 2008, ApJS, 178, 89

Doyle, A. P., Davies, G. R., Smalley, B., Chaplin, W. J., \& Elsworth, Y. 2014, MNRAS, 444, 3592

Dressing, C. D., Charbonneau, D., Dumusque, X., et al. 2015, ApJ, 800, 135

Eastman, J., Gaudi, B. S., \& Agol, E. 2013, PASP, 125, 83

Egeland, R., Soon, W., Baliunas, S., et al. 2017, ApJ, 835, 25

Feroz, F., Hobson, M. P., \& Bridges, M. 2009, MNRAS, 398, 1601

Foreman-Mackey, D., Hogg, D. W., Lang, D., \& Goodman, J. 2013, PASP, 125,306

Fossati, L., Marcelja, S. E., Staab, D., et al. 2017, A\&A, 601, A104

Fuhrmann, K., Axer, M., \& Gehren, T. 1993, A\&A, 271, 451

Fuhrmann, K., Axer, M., \& Gehren, T. 1994, A\&A, 285, 585

Fuhrmann, K., Pfeiffer, M., Frank, C., Reetz, J., \& Gehren, T. 1997a, A\&A, 323, 909

Fuhrmann, K., Pfeiffer, M. J., \& Bernkopf, J. 1997b, A\&A, 326, 1081

Fulton, B. J., Petigura, E. A., Howard, A. W., et al. 2017, AJ, in press, arXiv: 1703.10375

Gandolfi, D., Alcalá, J. M., Leccia, S., et al. 2008, ApJ, 687, 1303

Gandolfi, D., Barragán, O., Hatzes, A. P., et al. 2017, arXiv:1706.02532

Gray, R. O., \& Corbally, C. J. 1994, AJ, 107, 742

Grunblatt, S. K., Howard, A. W., \& Haywood, R. D. 2015, ApJ, 808, 127

Guenther, E. W., Barragan, O., Dai, F., et al. 2017, A\&A, in press, arXiv:1705. 04163

Gustafsson, B., Edvardsson, B., Eriksson, K., et al. 2008, A\&A, 486, 951 
Hatzes, A. P., Fridlund, M., Nachmani, G., et al. 2011, ApJ, 743, 75

Hauschildt, P. H., Allard, F., Ferguson, J., Baron, E., \& Alexander, D. R. 1999, ApJ, 525, 871

Haywood, R. D., Collier Cameron, A., Queloz, D., et al. 2014, MNRAS, 443, 2517

Haywood, R. D., Collier Cameron, A., Unruh, Y. C., et al. 2016, MNRAS, 457,3637

Henry, G. W. 1999, PASP, 111, 845

Hirano, T., Fukui, A., Mann, A. W., et al. 2016, ApJ, 820, 41

Howard, A. W., Sanchis-Ojeda, R., Marcy, G. W., et al. 2013, Natur, 503, 381 Isaacson, H., \& Fischer, D. 2010, ApJ, 725, 875

Jones, E., Oliphant, T., Peterson, P., et al. 2001, SciPy: Open Source Scientific Tools for Python, https://www.scipy.org/citing.html

Kipping, D. M. 2010, MNRAS, 408, 1758

Kobayashi, N., Tokunaga, A. T., Terada, H., et al. 2000, Proc. SPIE, 4008, 1056

Kovács, G., Zucker, S., \& Mazeh, T. 2002, A\&A, 391, 369

Kreidberg, L. 2015, PASP, 127, 1161

Kupka, F., \& Ryabchikova, T. A. 1999, POBeo, 65, 223

Kurucz, R. L. 2013, ATLAS1: Opacity Sampling Model Atmosphere Program, Astrophysics Source Code Library, ascl:1303.024

Lee, E. J., \& Chiang, E. 2017, ApJ, 842, 40

Liddle, A. R. 2007, MNRAS, 377, L74

Lindegren, L., \& Dravins, D. 2003, A\&A, 401, 1185

Lomb, N. R. 1976, Ap\&SS, 39, 447

Lopez, E. D. 2016, MNRAS, submitted, arXiv:1610.01170

Lopez, E. D., \& Fortney, J. J. 2014, ApJ, 792, 1

López-Morales, M., Haywood, R. D., Coughlin, J. L., et al. 2016, AJ, 152, 204

Lundkvist, M. S., Kjeldsen, H., Albrecht, S., et al. 2016, NatCo, 7, 11201

McQuillan, A., Mazeh, T., \& Aigrain, S. 2014, ApJS, 211, 24

Morton, T. D. 2015, Isochrones: Stellar Model Grid Package, Astrophysics Source Code Library, ascl:1503.010

Nelson, B. E., Ford, E. B., Wright, J. T., et al. 2014, MNRAS, 441, 442

Newville, M., Stensitzki, T., Allen, D. B., \& Ingargiola, A. 2014, LMFIT: NonLinear Least-Square Minimization and Curve-Fitting for Pythonú

Ofir, A. 2014, A\&A, 561, A138
Owen, J. E., \& Wu, Y. 2013, ApJ, 775, 105

Owen, J. E., \& Wu, Y. 2017, ApJ, 847, 29

Pepe, F., Cameron, A. C., Latham, D. W., et al. 2013, Natur, 503, 377

Pepe, F., Mayor, M., Rupprecht, G., et al. 2002, Msngr, 110, 9

Piskunov, N., \& Valenti, J. A. 2017, A\&A, 597, A16

Piskunov, N. E., Kupka, F., Ryabchikova, T. A., Weiss, W. W., \& Jeffery, C. S. 1995, A\&AS, 112, 525

Rappaport, S., Sanchis-Ojeda, R., Rogers, L. A., Levine, A., \& Winn, J. N. 2013, ApJL, 773, L15

Rogers, L. A. 2015, ApJ, 801, 41

Sanchis-Ojeda, R., Rappaport, S., Winn, J. N., et al. 2013, ApJ, 774, 54

Sanchis-Ojeda, R., Rappaport, S., Winn, J. N., et al. 2014, ApJ, 787, 47

Sanchis-Ojeda, R., Winn, J. N., Dai, F., et al. 2015, ApJL, 812, L11

Scargle, J. D. 1982, ApJ, 263, 835

Schwarz, U. J. 1978, A\&A, 65, 345

Scott, N. J., Howell, S. B., \& Horch, E. P. 2016, Proc. SPIE, 9907, 99072R

Sinukoff, E., Howard, A. W., Petigura, E. A., et al. 2017, AJ, in press, arXiv: 1705.03491

Smith, A. M. S., Cabrera, J., Csizmadia, S., et al. 2017, MNRAS, in press, arXiv: 1707.04549

Sneden, C. 1973, ApJ, 184, 839

Torres, G., Andersen, J., \& Giménez, A. 2010, A\&ARv, 18, 67

Valenti, J. A., \& Fischer, D. A. 2005, ApJS, 159, 141

Valenti, J. A., \& Piskunov, N. 1996, A\&AS, 118, 595

Vanderburg, A., Becker, J. C., Buchhave, L. A., et al. 2017, AJ, in press, arXiv: 1710.00026

Vanderburg, A., \& Johnson, J. A. 2014, PASP, 126, 948

Vanderburg, A., Plavchan, P., Johnson, J. A., et al. 2016, MNRAS, 459,3565

von Braun, K., Boyajian, T. S., ten Brummelaar, T. A., et al. 2011, ApJ, 740,49

Weiss, L. M., Rogers, L. A., Isaacson, H. T., et al. 2016, ApJ, 819, 83

Winn, J. N., Sanchis-Ojeda, R., Rogers, L., et al. 2017, arXiv:1704.00203

Yee, S. W., Petigura, E. A., \& von Braun, K. 2017, ApJ, 836, 77

Zeng, L., Sasselov, D. D., \& Jacobsen, S. B. 2016, ApJ, 819, 127 